\title{
Long non-coding RNA AC122108.1 promotes lung adenocarcinoma brain metastasis and progression through the Wnt/ $\beta$-catenin pathway by directly binding to aldolase $A$
}

\author{
Shaobin Feng ${ }^{1}$, Huiling Liu ${ }^{2}$, Peng Du ${ }^{3}$, Xushuai Dong ${ }^{1}$, Qi Pang $^{1}$, Hua Guo ${ }^{1}$ \\ ${ }^{1}$ Department of Neurosurgery, Shandong Provincial Hospital, Cheeloo College of Medicine, Shandong University, Jinan, China; ${ }^{2}$ Department of \\ Otolaryngology-Head and Neck Surgery, Shandong Provincial ENT Hospital, Cheeloo College of Medicine, Shandong University, Jinan, China; \\ ${ }^{3}$ Department of Neurosurgery, Shandong Provincial Hospital Affiliated to Shandong First Medical University, Jinan, China \\ Contributions: (I) Conception and design: H Guo; (II) Administrative support: None; (III) Provision of study materials or patients: S Feng; (IV) \\ Collection and assembly of data: S Feng, X Dong; (V) Data analysis and interpretation: S Feng, P Du; (VI) Manuscript writing: All authors; (VII) \\ Final approval of manuscript: All authors. \\ Correspondence to: Hua Guo. Department of Neurosurgery, Shandong Provincial Hospital, Cheeloo College of Medicine, Shandong University, Jinan \\ 250021, China. Email: drguohua@163.com.
}

\begin{abstract}
Background: Brain metastasis (BM) is a major pathological subtype of lung adenocarcinoma (LAD), but the pathogenic mechanisms of BM remain unclear. The potential prognostic biomarkers and therapeutic targets for BM of LAD urgently need to be identified. AC122108.1 is a recently discovered new long noncoding ribonucleic acid (RNA).

Methods: AC122108 was found to be overexpressed in a LAD BM cell model, and upregulated in $64.52 \%$ of LAD BM tissues. AC122108 is an independent factor of BM during LAD development; however, the molecular mechanisms and clinical significance of AC122108.1 in LAD have not yet been established. Additionally, in vitro and in vivo experiments showed that the direct binding of AC122108.1 with aldolase A (ALDOA) enhanced the proliferation, apoptosis, invasiveness, migration, and metastasis of LAD cells.

Results: This RNA-protein complex decreased the stability of the $\beta$-catenin destruction complex, leading to the accumulation of $\beta$-catenin in the cytoplasm and ultimately its translocation into the nucleus to activate Wnt(wingless/integrated)/ $\beta$-catenin signaling.

Conclusions: Overall, AC122108.1 promotes LAD BM and its progression through the Wnt/ $\beta$-catenin pathway by directly binding to ALDOA. This study provides insights into the regulatory mechanism of the LAD BM. AC122108.1 may serve as a potential therapeutic target and prognostic biomarker of LAD.
\end{abstract}

Keywords: Lung adenocarcinoma (LAD); brain metastasis; AC122108.1; ALDOA; $\beta$-catenin

Submitted Sep 28, 2021. Accepted for publication Dec 06, 2021.

doi: 10.21037/atm-21-5707

View this article at: https://dx.doi.org/10.21037/atm-21-5707

\section{Introduction}

Non-small cell lung cancer (NSCLC) is the most common primary tumor, and accounts for approximately $85 \%$ of all lung cancer cases (1-3). Lung adenocarcinoma (LAD) constitutes approximately half of NSCLCs and accounts for the largest proportion of single metastases in the brain (1-3). The brain metastasis (BM) of lung cancer is the most common subtype of intracranial metastases in adults, and seriously affects the prognosis of patients (1). There have been significant improvements in major therapeutic strategies, including microsurgery, chemotherapy, whole brain radiotherapy, and stereotactic radiosurgery, in recent decades; however, BM in LAD is associated with a poor prognosis (4-7). It is thought that unidentified molecules and molecular mechanisms might play key roles in the development of aggressive LAD. Exploring new targets and 
identifying key biomarkers would help ensure that patients suffering from LAD BM are provided with an effective and safe diagnosis and treatment.

Long non-coding ribonucleic acids (lncRNAs), which are a subset of regulatory RNAs longer than 200 nucleotides that lack a protein-coding function, play indispensable roles in many fundamental biological mechanisms and pathological processes $(8,9)$. LncRNA also has biological functions in tumor immunology, including tumor antigen presentation, immune escape and immune cell infiltration (10). LncRNA is becoming a new biomarker for predicting the prognosis of various cancers, including HOTAIR in lung cancer (11). In this study, we focused on the key roles of lncRNAs in cancer metastasis and progression, especially in $\operatorname{LAD} B M(7,12,13)$.

In this study, a second-generation sequencing analysis identified lncRNA AC122108.1, which is located on chromosome 15 , has a length of $424 \mathrm{bp}$, and is highly expressed in the BM of LAD. Notably, this is the first study to identify and functionally characterize AC122108.1 (14). We found that the expression of AC122108.1 appeared to correlate with LAD metastases and that a high level of AC122108.1 indicated a poor prognosis.

Aldolase A (ALDOA), which has been reported to serve as a diagnostic and prognostic marker, contributes to cell proliferation and promotes lung cancer cell metastasis $(15,16)$. Previous lung cancer reports have shown that $\mathrm{Wnt} / \beta$-catenin signaling plays a key role in lung cancer development and progression and is one of the most effective cascades modulating tumor invasion and metastasis (17). Several components of $\mathrm{Wnt} / \beta$-catenin pathway and $\beta$-catenin target genes, including $\mathrm{c}-\mathrm{Myc}$, cell Cyclin D1, VEGF-A, MMP-7 and survivin are overexpressed in lung adenocarcinoma (18). Abnormal activation of $\beta$-catenin signal is also involved in epithelialmesenchymal transition (EMT), which is a key step in the process of tumor metastasis (19). A study showed that $\beta$-catenin knockdown inhibited metastatic potential, including migration and invasion, and inhibited the brain metastasis of PC14PE6/LvBr4 cells in vivo (20). Further, our results indicated that AC122108.1 is downregulated in PC14/B cells, a highly brain metastatic cell line of LAD, which significantly suppressed the proliferative, invasive, and metastatic potential of these cells $(21,22)$. The results of in vivo and in vitro experiments demonstrated that AC122108.1 plays an important role in regulating the proliferation, cell cycle, apoptosis, invasiveness, migration, and metastasis of LAD. Specifically, we hypothesized that
AC122108.1 binds to ALDOA and is involved in Wnt/ $\beta$-catenin signaling, which promotes the metastasis and progression of LAD, and that AC122108 could provide a new therapeutic perspective on how to target LAD BM. We present the following article in accordance with the ARRIVE reporting checklist (available at https://dx.doi. org/10.21037/atm-21-5707).

\section{Methods}

\section{Cell culture}

The human LAD cell line, PC14, and its sub-clone, PC14/B, which have significant tendency toward $\mathrm{BM}$, were obtained from the Committee of the Type Culture Collection of the Chinese Academy of Sciences (Shanghai, China). The cells were cultured at $37{ }^{\circ} \mathrm{C}$ with $5 \%$ carbon dioxide in Dulbecco's Modified Eagle Medium (DMEM; Gibco, Thermo Fisher Scientific, USA) containing 10\% fetal bovine serum (FBS; Biological Industries, USA) and 1\% penicillin/streptomycin.

\section{Clinical specimens and patients}

All procedures performed in this study involving human participants were in accordance with the Declaration of Helsinki (as revised in 2013). All aspects of this study were approved by the Research Ethics Committee of Shandong Provincial Hospital Affiliated with Shandong University (SWYX: No. 2019-168). Eighty-seven histologically confirmed LAD tissue samples and 19 normal lung and brain tissue samples were collected during surgical procedures from January 2014 to January 2019 with complete follow-up data. Written consent was obtained from all patients or their families.

\section{RNA extraction, RNA sequencing, and data analysis}

Total lung cancer cell RNA was extracted by TRIzol reagent (Invitrogen, Carlsbad, CA, USA), and the integrity and concentration of the samples were then determined using an Agilent 2100 RNA Nano 6000 Assay Kit (Agilent Technologies, CA, USA). Transcriptome sequencing was performed by Beijing Annoroad Gene Technology Co. (Beijing, China, www. annoroad.com/) using the Illumina HiSeq Xten platform in PE150 mode (Illumina, Inc., San Diego, CA, USA). Briefly, a total of 106,830 messenger RNAs (mRNAs) and 81999 known lncRNAs were identified 
from the most reliable databases, including NONCODE, Ensembl, and Gencode, and other relevant literature. The differential expression of mRNAs and lncRNAs was defined using $\mathrm{P}$ value and $\log$ fold change. Additionally, the Gene Set Enrichment Analysis (GSEA), Gene Ontology (GO) (www.geneontology.org), and Kyoto Encyclopedia of Genes and Genomes (KEGG) databases (http://www.genome. ad.jp/kegg/) were used to identify potential target genes and pathways in the mRNA expression profile. The lower the $\mathrm{P}$ value, the higher the significance; a threshold of $\mathrm{P}<0.05$ was used.

\section{$R N A$ isolation and $q R T-P C R$}

Total RNA was extracted from tissues or cultured cells with TRIzol reagent (Invitrogen, NY, USA). One microgram of total RNA was reverse transcribed into complementary deoxyribonucleic acid (cDNA) using random primers under standard conditions with Reverse Transcription Kits (Takara, Dalian, China). Real-time polymerase chain reaction (RTPCR) assays of RNA expression levels were carried out using a SYBR Green PCR Kit (Takara) in accordance with the manufacturer's instructions. The results were normalized to the expression of glyceraldehyde-3-phosphate dehydrogenase (GAPDH), and the data were analyzed using the comparative cycle threshold (CT; $2^{-\Delta \Delta C T}$ ) method. The relative sequences used for the PCR are shown in Table S1.

\section{Cell transfection}

All the small-interfering RNAs (siRNAs) and lentiviruses were constructed and synthesized by GeneChem (Shanghai, China). For RNA interference, 4 different siRNAs that targeted ALDOA RNA were designed to decrease the off-target effects. PC14/B cells were seeded in 6-well plates and incubated overnight, and the target cells were then transfected with different siRNAs by Lipofectamine 3000 (Invitrogen). After testing and verification, the most effective siRNA for ALDOA knockdown was used in subsequent assays.

\section{Cell proliferation assay}

The cell viability rate was assessed using a Cell Counting Kit-8 (CCK-8) kit (Dojindo, Kumamoto, Japan). In brief, after transfection, the cells were plated into 96well plates with $100 \mu \mathrm{L}$ per well of DMEM supplemented with $10 \%$ FBS at a density of 1,500 cells per well for
96 h. Each group was tested with 5 replicates. The medium was replaced with $90 \mu \mathrm{L}$ of fresh DMEM mixed with $10 \mu \mathrm{L}$ of CCK-8 solution at a series of time points $(0,24,36,48,72$, and $96 \mathrm{~h})$, and the absorbance was measured at $450 \mathrm{~nm}$. Cell viability was calculated in accordance with the manufacturer's instructions.

\section{Colony formation assay}

Different groups of cells (800 cells/well) were trypsinized into a single-cell suspension, seeded into 6-well plates, and cultured in a medium supplemented with $10 \%$ FBS. The medium was replaced every 3 days for 2 weeks. The cells were fixed with $4 \%$ paraformaldehyde for $30 \mathrm{~min}$, and then stained with $1 \%$ crystal violet for another $20 \mathrm{~min}$ on the 14th day. Clones containing more than 50 cells were counted and recorded by microscopy. Three independent replicates were performed.

\section{Flow cytometric analysis}

For the cell apoptosis analysis, the cells were washed in cold phosphate buffered saline (PBS) before staining with Annexin-V/fluorescein isothiocyanate (BD Biosciences, CA, USA) and Propidium Iodide (PI) to determine apoptosis, and the ratio of viable, early apoptotic, apoptotic and dead cells was analyzed using flow cytometry. The relative data were analyzed using FlowJo software 7.0 (Tree Star Inc., Ashland, USA).

\section{Scratch assay}

For the cell scratch assays, the cells were cultured in 6-well plates with regular medium until they reached $80-90 \%$ confluence. After the cells were scratched with a straight line using a $20-\mu \mathrm{L}$ pipette tip, they were washed 3 times with PBS. The cells were cultured with serum-free medium, and images were recorded by inverted microscopy at 0 and $24 \mathrm{~h}$.

\section{Cell migration and invasion assays}

Cell migration and invasion were measured by Transwell chamber culture plates (24-well format, 8- $\mu \mathrm{m}$ pore size, Corning Costar, Corning, NY, USA). Transwell chambers without Matrigel (BD, San Diego, CA, USA) were used to measure cell motility, while Transwell chambers coated with Matrigel were used to determine cell invasiveness. 
Cells $\left(2 \times 10^{4}\right.$ per well for cell migration, $3 \times 10^{4}$ cells per well for cell invasion) were resuspended in $100 \mu \mathrm{L}$ of serumfree medium, and the cell suspension was seeded into the top chamber. DMEM supplemented with $20 \%$ FBS was added to the lower chamber as a chemoattractant. After incubation at $37^{\circ} \mathrm{C}$ for $24 \mathrm{~h}$, the cells that remained on the upper side of the chamber were wiped with cotton swabs, while those that invaded through the pores were fixed in $4 \%$ paraformaldehyde and stained with $1.0 \%$ crystal violet. Images were taken by microscopy, and the cells were counted using ImageJ software (Bethesda, Maryland, USA).

\section{Western blot analysis}

Equal amounts of protein samples were collected using Radio Immunoprecipitation Assay (RIPA) lysis buffer and evaluated using a bicinchoninic acid assay (BCA) protein kit (Beyotime Biotechnology, China) in accordance with the manufacturer's instructions. Cell protein lysates were separated by $6-12 \%$ sodium dodecyl sulphate-polyacrylamide gel electrophoresis (SDSPAGE) and transferred onto polyvinylidene fluoride (PVDF) membranes (Millipore, Massachusetts, USA). The membranes were then incubated with specific antibodies at $4{ }^{\circ} \mathrm{C}$ overnight. The immunocomplexes were incubated with horseradish peroxidase-linked secondary antibodies at room temperature for $1 \mathrm{~h}$. Immunoreactive bands were detected with an enhanced chemiluminescence kit (Thermol Biotech Inc., USA), and visualized using an electrophoresis image analysis system (Bio-Rad, CA, USA).

The following antibodies were used: ALDOA (PTG, 11217-1-AP), $\beta$-Catenin (Affinity, AF6266), P- $\beta$-Catenin (Affinity, DF2989), GSK3 $\beta$ (PTG, 22104-1-AP), FLAG (PTG, 20543-1-AP), LEF1(PTG,14972-1-AP), Vimentin (Affinity, AF7013), N-Cadherin (PTG, 22018-1-AP), E-Cadherin (PTG, 20874-1-AP), Cyclin D1(PTG, 601861-lg), MMP7(PTG, 10374-2-AP), c-Myc (Affinity, AF0358), GAPDH (PTG, 60004-1-Ig), and $\beta$-actin (PTG, 60008-1$\mathrm{Ig}$ ). The antibodies were purchased from Proteintech (PTG, China) and Affinity Biosciences (Affinity, China).

\section{RNA-FISH assays}

Fluorescence in situ hybridization (FISH) assays were designed and performed using a FISH Kit (RiboBio, China) in accordance with the manufacturer's instructions. The cells were stained with AC122108.1, 18S, and U6 synthetic, Cy3-labelled probes for $12 \mathrm{~h}$ at $37^{\circ} \mathrm{C}$. The cells were then stained with 4',6-diamidino-2-phenylindole (Beyotime, China). Fluorescence detection was performed with a fluorescence microscope (Olympus, Japan).

\section{Animal models of BM}

All animal experiments were approved under a project license (No. 2019144) granted by the Institutional Animal Care and Use Committee of Shandong University and conducted in strict accordance with its recommendations and ethical guidelines for the care and use of animals. Sixteen 4-week-old BALB/c athymic mice were obtained from Vital River (Beijing, China) and randomly divided into the following two groups: the AC122108.1-knockdown group and the control group. To generate BMs, the mice were anaesthetized by isoflurane, and PC14/B cells $\left(1 \times 10^{6} / 50 \mu \mathrm{L}\right.$ DMEM) were then injected very slowly into the internal carotid artery under surgical visualization (23). After the operation, the animals were put back into cages and given free access to food and water. After 6 weeks, the mice were humanely sacrificed, and the presence of BMs was examined with a microscope after hematoxylin and eosin (HE) staining.

\section{RNA pulldown assay}

Biotin-labelled RNAs were incubated with cell lysates for $6 \mathrm{~h}$ at $4{ }^{\circ} \mathrm{C}$ with the MaxiScript T7 kit (Ambion). Next, streptavidin beads were added for component enrichment, and the proteins were tested with biomass spectrometry (Bio-MS; Thermo Orbitrap Fusion, USA) and silver staining (Solarbio, Beijing, China) according to standard protocols.

\section{RNA RIP assay}

The EZ-Magna RIP kit (Millipore, Billerica, MA) was used to perform the RNA immunoprecipitation (RIP) assay in accordance with the manufacturer's protocol. Briefly, the cells were lysed with RIP buffer, supplemented with a ribonuclease (RNase) inhibitor, followed by the target antibody. The RNA-protein complexes were enriched by protein $\mathrm{A} / \mathrm{G}$ beads, and finally, the precipitated RNAs were eluted and analyzed by quantitative RT-PCR (qRT-PCR).

\section{ECAR measurement}

LAD cells were plated in an XFe96 plate. Glycolysis was 
measured using a Seahorse Bioscience XFe96 Extracellular Flux Analyzer. After being slightly diluted with XFe96 medium, glucose $(10 \mathrm{mM})$, oligomycin $(1 \mu \mathrm{M})$, and 2-deoxy-glucose (2-DG, $50 \mathrm{mM}$ ) were injected into the storage chambers in proper order. The extracellular acidification rate (ECAR) was detected by the injections of the above components in turn into the experimental sample at the specified time point. The glycolytic capacity, glycolysis, and glycolytic reserve were obtained from this measurement. The dates were output by Wave software (Seahorse Bioscience, USA).

\section{co-IP assays}

The cells were lysed in immunoprecipitation (IP) lysis buffer with a protease inhibitor and RNase inhibitor. In brief, glycogen synthase kinase- 3 beta (GSK3 $\beta$-flag, Sino Biological, HG10044-NF) was incubated with FLAG M2 Beads (Sigma-Aldrich, M8823), and then incubated with the cleared supernatant of the cell lysates at $4{ }^{\circ} \mathrm{C}$ overnight. The magnetic beads were washed with buffer 4 times, collected and boiled, and the immunoprecipitated proteins were then analyzed by Western blotting.

\section{Immunobistochemistry and immunofluorescence assay}

The mouse brains were divided into 2 parts: half of the samples were collected and fixed in $10 \%$ formalin buffer, embedded in paraffin, and cut into slices to stain with $\mathrm{HE}$ according to standard protocols. The other half were cut into frozen sections for immunofluorescence staining. The avidin-biotin-peroxidase method was adopted to determine the location and relative expression level of the target proteins. The sections were visualized and recorded by fluorescence microscopy (Olympus, Japan).

\section{Statistical analysis}

Statistical analyses were performed using R software (V 3.6.1). The results are presented as the mean \pm standard error (SE). Statistical differences between two groups were determined using the Student's $t$-test, and an analysis of variance was used to determine the statistical differences between 3 or more groups based on 1 factor. The strength of association between the ranked variables was assessed using the Spearman's rank correlation test and $\chi^{2}$ test. Each experiment was replicated 3 times. All tests were 2-sided. A
$\mathrm{P}$ value $\leq 0.05$ was considered statistically significant.

\section{Results \\ AC122108.1 was significantly upregulated in the LAD cell lines and tissues and was related to LAD BM}

To identify novel lncRNAs involved in the development of LAD, we carried out differential mRNA and lncRNA expression analyses using a high-throughput screening assay. We analyzed differential gene expression and known lncRNAs in the PC14 human LAD cell line and its subclone PC14/B using Illumina HiSeq Xten in PE150 sequencing mode (Figure 1A). Based on the results, NONHSAT042195.2, a clone-based Ensembl gene that we named AC122108.1, was significantly more upregulated ( $>500$ fold) in the PC14/B cell line than the PC14 cell line (Figure 1B,1C). The qRT-PCR data from a cohort of 106 clinical samples confirmed the above results. Further, AC122108.1 expression was higher in brain metastatic LAD tissue than in primary LAD tissue $(3.62$ fold; $\mathrm{P}<0.01)$ and non-brain metastatic LAD tissue $(2.84$ fold; $\mathrm{P}<0.05)$. In addition, we divided 87 patients into 3 groups depending on their pathological stage and the sites of distant metastasis and analyzed the relative expression levels of AC122108.1 by qRT-PCR. We found that AC122108.1 expression was higher in brain metastatic LAD tissue than in matched adjacent normal tissue and even other non-brain metastatic LAD tissues (Figure 1D,1E). The results provided further evidence of the potential role of AC122108.1 in LAD BM.

\section{$A$ high level of AC122108.1 was associated with a poor prognosis in LAD patients}

The median follow-up period with patients in this study was 40 months. First, a preliminary investigation of the survival rate of the 87 LAD patients was performed. $9.2 \%$ of primary, $17.2 \%$ of non-brain metastatic, and $24.1 \%$ of brain metastatic LAD patients died, and the overall survival (OS) of the brain metastatic LAD patient group was obviously lower than that of the 2 other groups $(\mathrm{P}=0.009$; Figure $1 F)$. Next, to further understand the association between OS and the expression of AC122108.1, we regrouped all the LAD patients. 43 of the 87 patients included in the investigation (49.4\%) died. Of the patients who died, 34 (39.1\%) were from the high-level AC122108.1 group and 9 (10.3\%) were from the low-level AC122108 group. The OS of the LAD patients with a high expression level of AC122108.1 


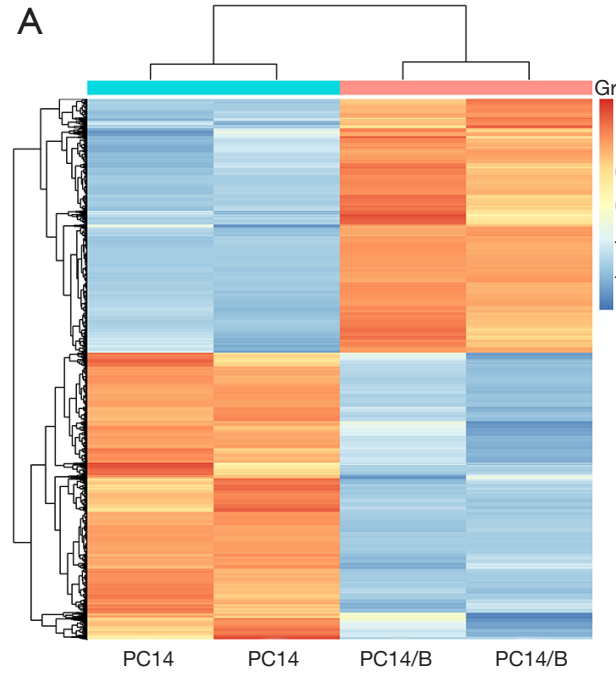

$E$

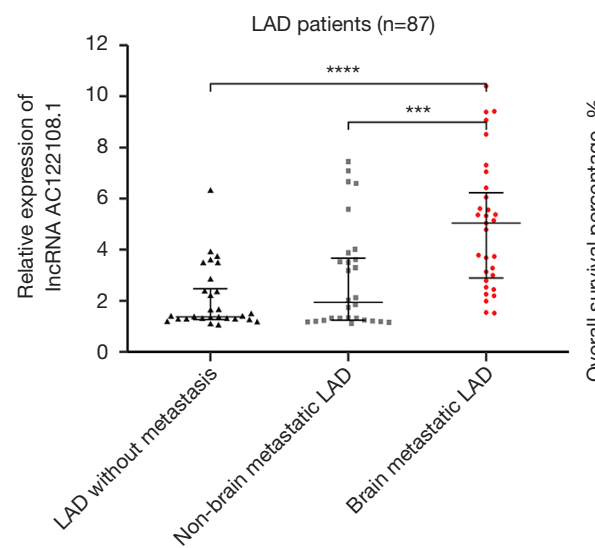

B

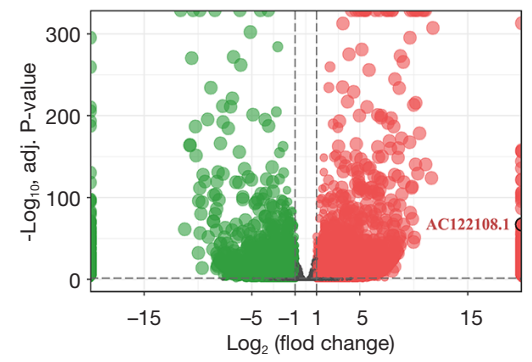

C

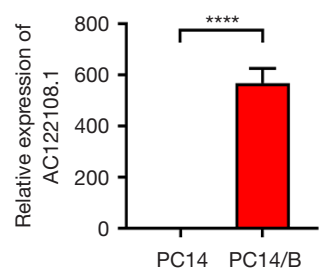

LAD patients $(n=87)$

$\mathrm{F}$

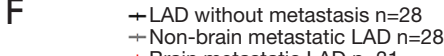
+Brain metastatic LAD $n=31$

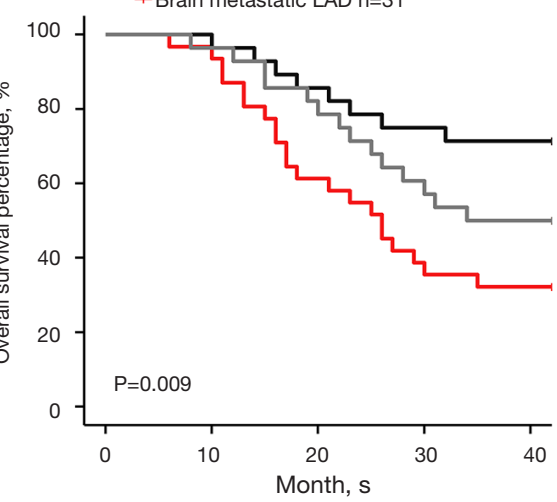

$\mathrm{D}$
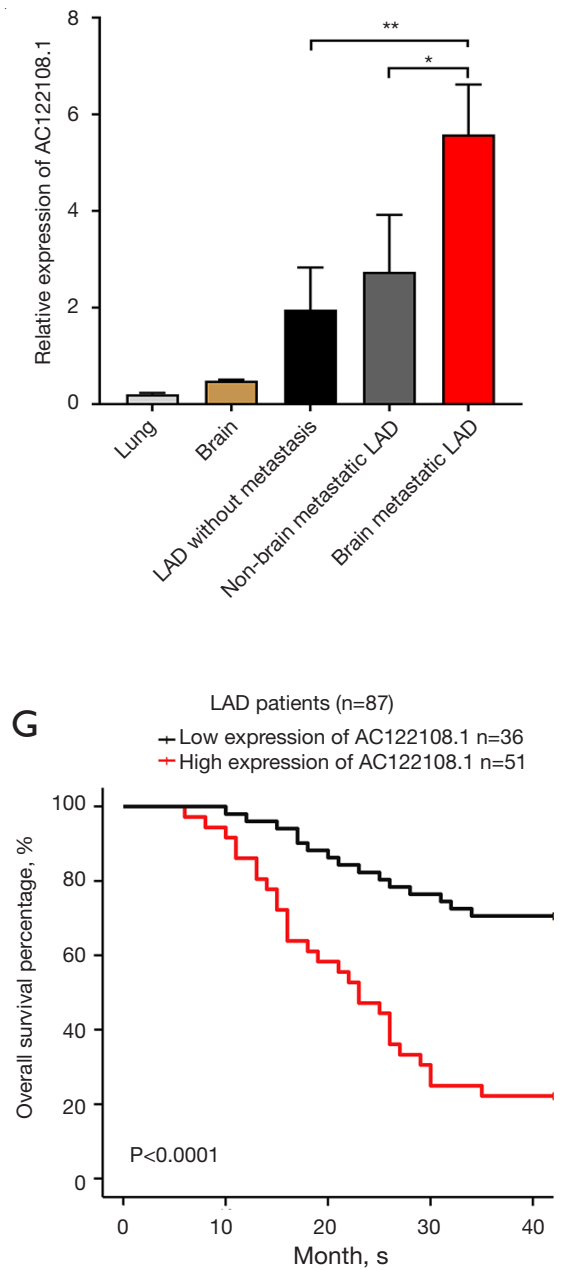

Figure 1 AC122108.1 is highly expressed in the LAD cell lines and thus has prognostic value. (A) A heat map showing the hierarchical clustering of differentially expressed lncRNAs in PC14/B compared to PC14. (B) The expression level of lncRNAs, including AC122108.1, was visualized by a volcano map. (C) AC122108.1 expression level in LAD cell lines. (D) The expression of AC122108.1 in different groups of patients' tissues. (E) The endogenous expression levels of AC122108.1 in 87 clinical LAD specimens classified into 3 teams were determined by the diagnosis standards of the Union for International Cancer Control (UICC) and the existence of BMs. (F) The OS of the 87 LAD patients was recorded based on the above diagnostic criteria. (G) The OS of the 87 LAD patients was recorded based on the expression levels of AC122108.1 $\left.{ }^{*} \mathrm{P}<0.05,{ }^{* *} \mathrm{P}<0.01,{ }^{* * *} \mathrm{P}<0.001,{ }^{* * * *} \mathrm{P}<0.0001\right)$. LAD, lung adenocarcinoma; $\mathrm{BM}$, brain metastasis; OS, overall survival.

was significantly lower than that of patients with a low expression level of AC122108.1 ( $\mathrm{P}<0.001$; Figure $1 G)$.

To elucidate how AC122108.1 is involved in the BM of LAD development, we analyzed the correlation of AC122108.1 expression with clinicopathological factors. We found a significant correlation between AC122108.1 expression and tumor $(\mathrm{T})$, node $(\mathrm{N})$, and metastasis $(\mathrm{M})$ staging $(\mathrm{P}=0.003)$ and expressions of Ki-67 $(\mathrm{P}<0.001$; Table 1). However, no statistically significant differences were found in terms of age, sex, or tumor size. AC122108.1 expression was a predictive factor of BM during LAD development (Table 2). Thus, these results provided further evidence that AC122108.1 could be used as a potential biomarker to evaluate the prognosis of LAD patients. 
Table 1 Demographic characteristics of the overall LAD population

\begin{tabular}{|c|c|c|c|c|c|}
\hline Factor & Total (\%) & \multicolumn{2}{|c|}{ AC122108.1 expression } & R-square & $P$ value \\
\hline All case & 87 & $51(58.6)$ & $36(41.4)$ & & \\
\hline Age at surgery, years & & & & 0.451 & 0.198 \\
\hline$<60$ & $47(54.0)$ & $31(60.8)$ & $16(44.4)$ & & \\
\hline Sex & & & & 0.869 & 0.702 \\
\hline Male & $45(51.7)$ & $25(49.0)$ & $20(55.6)$ & & \\
\hline Female & $42(48.3)$ & $26(51.0)$ & $16(44.4)$ & & \\
\hline TNM (UICC 2017) & & & & 0.184 & 0.003 \\
\hline $\mathrm{TN}_{\mathrm{x}} \mathrm{M}_{1}$ (with brain metastasis) & $31(35.6)$ & $11(21.6)$ & $20(55.6)$ & & \\
\hline Tumor size & & & & 0.125 & 0.080 \\
\hline$\leq 3 \mathrm{~cm}$ & $32(36.8)$ & $23(45.1)$ & $9(25.0)$ & & \\
\hline $3-5 \mathrm{~cm}$ & $14(16.1)$ & $9(17.6)$ & $5(13.9)$ & & \\
\hline$>5 \mathrm{~cm}$ & $41(47.1)$ & $19(37.3)$ & $22(61.1)$ & & \\
\hline Ki-67 & & & & 0.058 & $<0.001$ \\
\hline$\leq 10 \%$ & $25(28.7)$ & $23(45.1)$ & $2(5.6)$ & & \\
\hline $10-30 \%$ & $15(17.3)$ & $12(23.5)$ & $3(8.3)$ & & \\
\hline
\end{tabular}

LAD, lung adenocarcinoma; TNM, tumor node metastasis; Ki-67, proliferation marker protein Ki-67.

Table 2 Univariate and multivariate analysis of LAD

\begin{tabular}{|c|c|c|c|c|c|c|}
\hline Variables & \multicolumn{3}{|c|}{ Univariate analysis } & \multicolumn{3}{|c|}{ Multivariate analysis } \\
\hline Age at surgery & 2.26 & $1.225-4.178$ & 0.009 & 2.22 & $1.068-4.599$ & 0.033 \\
\hline Sex & 1.15 & $0.631-2.094$ & 0.648 & 1.49 & $0.740-3.004$ & 0.263 \\
\hline TNM grade & 1.80 & $1.221-2.657$ & 0.003 & 1.42 & $0.901-2.251$ & 0.131 \\
\hline Ki-67 & 2.65 & $1.650-4.269$ & 0.000 & 1.56 & $0.881-2.769$ & 0.127 \\
\hline AC122108.1 & 1.32 & $1.193-1.466$ & 0.000 & 1.30 & $1.090-1.539$ & 0.003 \\
\hline
\end{tabular}

$\mathrm{LAD}$, lung adenocarcinoma; HR, hazard ratio; $\mathrm{Cl}$, confidence interval. 


\section{The knockdown of AC122108.1 affected LAD cell proliferation, apoptosis, migration, and invasion}

We examined the effects of AC122108.1 knockdown on the behaviors of the LAD cell lines, and found that the expression of AC122108.1 was downregulated in PC14/B cells, and the rate of LAD cell proliferation was obviously reduced compared to that of $\mathrm{PC} 14$ cell proliferation (Figure 2A). Additionally, compared to that of the PC14 cells, the colony formation ability of the PC14/B cells was markedly suppressed by the knockdown of AC122108.1 (Figure 2B,2C). The flow cytometry results showed that the knockdown of AC122108.1 promoted cell apoptosis to a certain extent (Figure 2D,2E). Further, the low expression of AC122108.1 significantly suppressed the migration and invasion of PC14/B cells (Figure 2F-2K). Together, these results indicate that the knockdown of AC122108.1 more strongly inhibited the tumorigenicity of PC14/B cells than that of PC14 cells.

\section{AC122108.1 interacted with ALDOA}

Next, we aimed to investigate the mechanism by which AC122108.1 regulates the behaviors of LAD cells. The functions of IncRNAs are greatly affected by their subcellular localization. First, we identified the subcellular location of AC122108.1 in PC14/B cells by RNA FISH assays. U6 and $18 \mathrm{~S}$ were used as the internal controls, and we found that the signals of AC122108.1 were distributed in the cytoplasm and nucleus of the PC14/B cells (Figure $3 A$ ). Further, as has been shown in many previous studies, many lncRNAs exert their biological functions by physically interacting with proteins. To investigate the possible molecular mechanism by which AC122108.1 plays a role in $\mathrm{LAD}$ cells, we conducted pulldown experiments and a mass spectrometry (MS) analysis to identify the intracellular binding proteins of AC122108. We selected potential interacting proteins from the MS library, and biotinylated AC122108.1 and its antisense RNA, were incubated with cell lysates of PC14/B cells, after which another pulldown experiment was conducted.

ALDOA was identified as a potential RNA binding protein $(\mathrm{RBP})$, which was further verified by immunoblotting assay (Figure 3B,3C) and PAGE silver staining (Figure 3D). 3 protein bands (bands 1-3 numerically labelled on the gel, and indicated with arrows in Figure 3D) again identified ALDOA as a putative interacting protein.

To determine whether AC122108.1 specifically binds to RBPs, we next investigated the interaction between AC122108.1 and ALDOA by a RNA pulldown assay followed by immunoblotting and RIP assays (Figure 3B-3E). Our results showed that ALDOA specifically interacted with AC122108.1. Further, to identify the intramolecular regions of AC122108.1 that interacted with ALDOA, 6 fragments of AC122108.1 (1-124, 1-224, 1-324, 125-424, 225-424, and 325-424 nt) were transcribed in vitro and biotinylated. The fragments were then used in pulldown assays, and we found that segment 1-124 nt of AC122108.1 interacted with ALDOA (Figure 3F-3H).

Additionally, the 3-dimensional (3D) structure prediction of RNA-protein complexes successfully predicted that these 2 binding fragments contained stable RNA-protein structures, according to the corresponding energy calculated as the root-mean-square-deviation-based score, which provided further evidence that the aforementioned predicted AC122108.1 region interacted with ALDOA (Figure 3I-37). In addition, the RNA-protein example in Figure $3 I$ (No. 5) may be the most stable structure with the largest probability and smallest energy. Taken together, these data suggest that ALDOA directly binds to AC122108.1, and the 2 molecules can be integrated into an RNA-protein complex to perform their biological functions.

\section{The knockdown of ALDOA and AC122108.1 affected the expression of $L A D$ cell proliferation, apoptosis, migration and invasion}

To examine the effects of ALDOA on the LAD cell lines PC14 and PC14/B behaviors, ALDOA gene interference sequence (si-ALDOA) and ALDOA gene overexpression sequence (oe-ALDOA) were used to generate ALDOAknockdown and overexpression in PC14 and PC14/B cells, respectively. Compared to those formed by si-vector cells, the numbers of colonies formed by the generated siALDOA cells were significantly decreased, while the oeALDOA colony numbers were increased (Figure $4 A, 4 B$ ). siALDOA also induced cell apoptosis, while oe-ALDOA had the opposite effect (Figure 4C,4D). Further, the migration and invasion capabilities of the cells were obviously enhanced by oe-ALDOA, and reduced by si-ALDOA (Figure 4E-4f).

Co-transfections were also performed to evaluate the interaction between AC122108.1 and ALDOA. As Figure 4 shows, ALDOA functions as an oncogene in the LAD cell lines, and the knockdown of AC122108.1 reduces the tumorigenicity of ALDOA. 


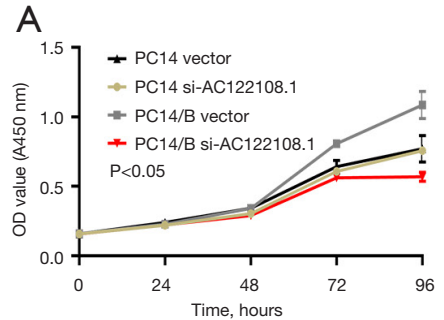

B
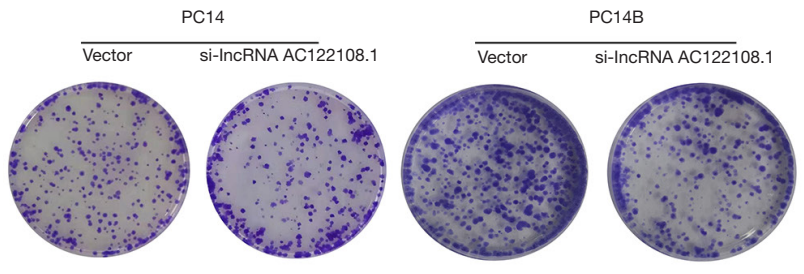

D

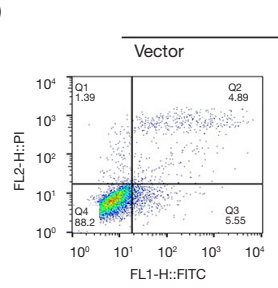

PC14

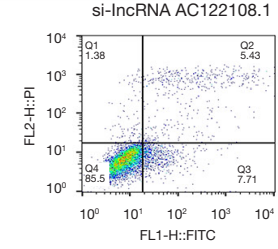

PC14

$\mathrm{F}$
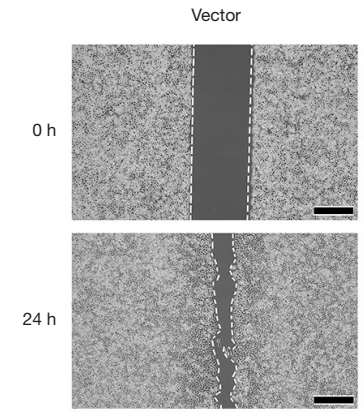

$\mathrm{H}$
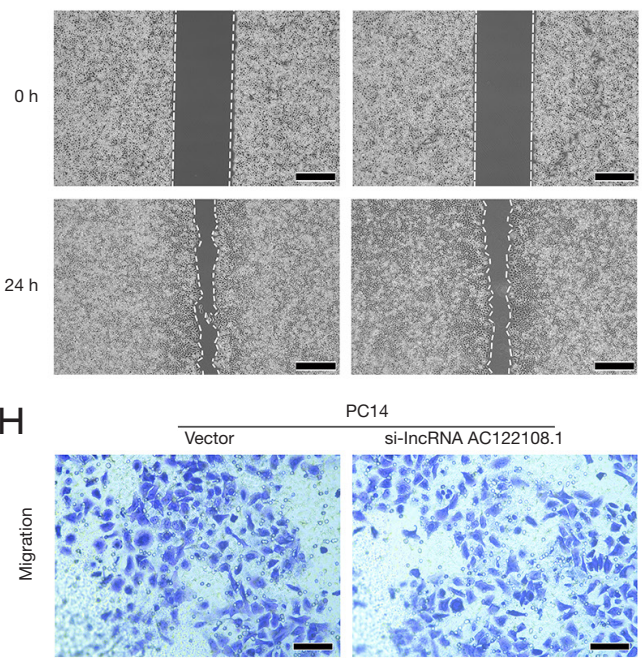

PC14
si-IncRNA AC122108.1

J

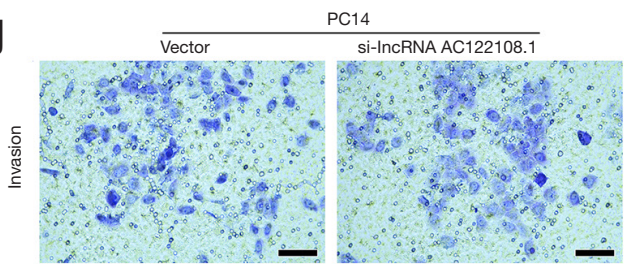

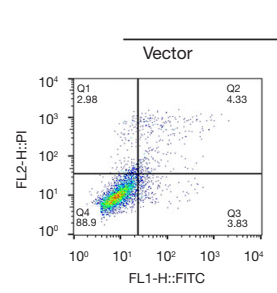

PC14B

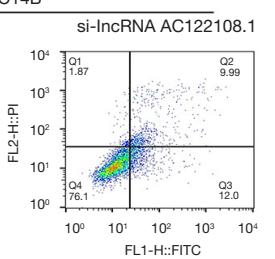

PC14B

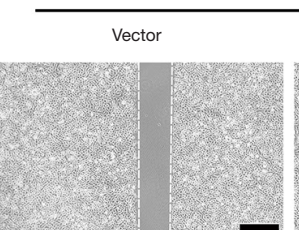

si-IncRNA AC122108.1
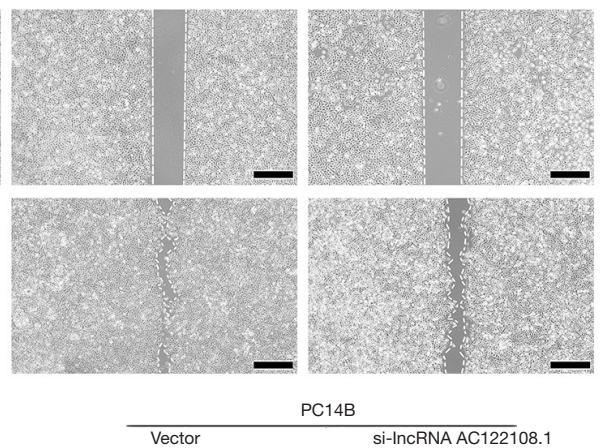

$\mathrm{PC} 14 \mathrm{~B}$


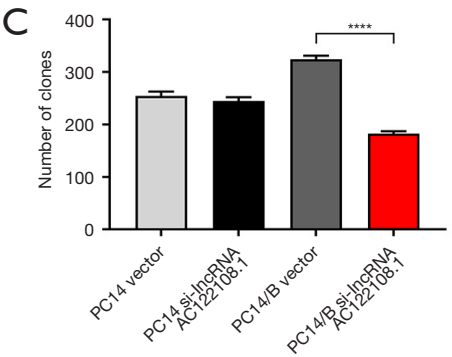

E

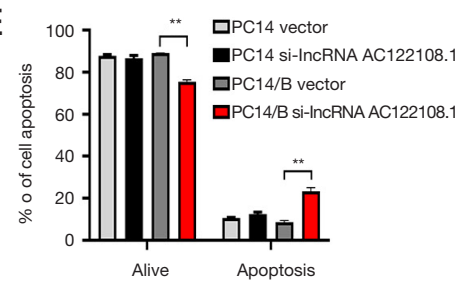

G
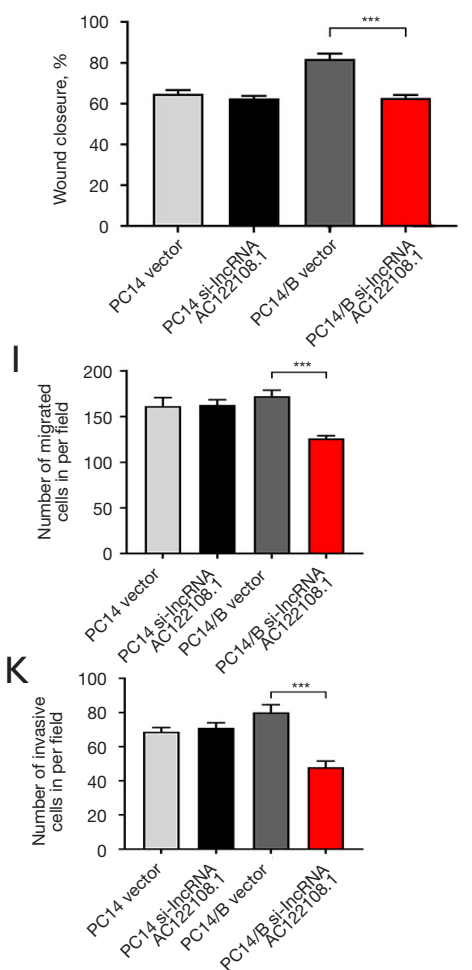

Figure 2 The effect of AC122108.1 knockdown (si-IncRNA AC122108.1) on LAD cell proliferation, cell cycle, apoptosis, migration, and invasion. (A) CCK-8 assay of the proliferation of PC14 and PC14/B. (B,C) Clone formation ability of PC14 and PC14/B after AC122108.1 silencing (crystal violet staining). (D,E) Cell apoptosis of PC14 and PC14/B after AC122108.1 silencing. (F,G) Wound healing assay of PC14 and PC14/B after AC122108.1 silencing (scale bar: $200 \mu \mathrm{m}$ ). (H,I) Cell migration assay of PC14 and PC14/B after AC122108.1 silencing (crystal violet staining, scale bar: $25 \mu \mathrm{m}$ ). (J,K) cell invasion assay of PC14 and PC14/B after AC122108.1 silencing (crystal violet staining, scale bar: $\left.25 \mu \mathrm{m} ;{ }^{* *} \mathrm{P}<0.01,{ }^{* * *} \mathrm{P}<0.001,{ }^{* * * *} \mathrm{P}<0.0001\right)$. LAD, lung adenocarcinoma. 
A

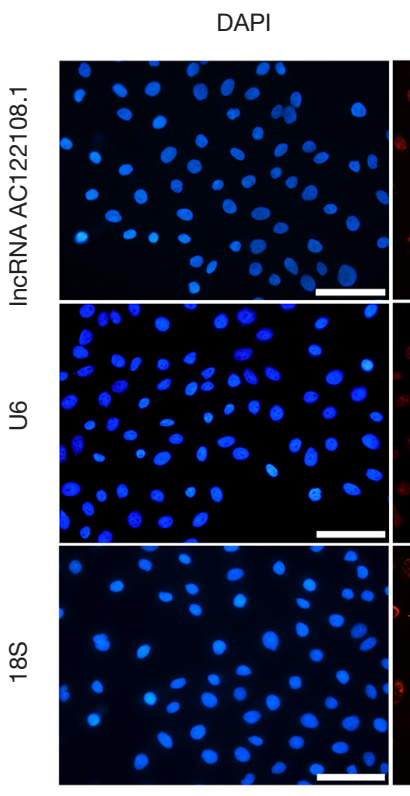

$E$

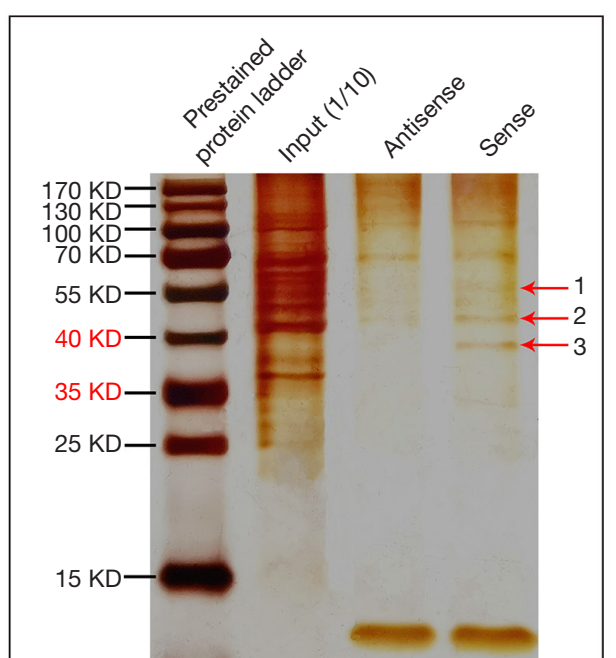

Суз

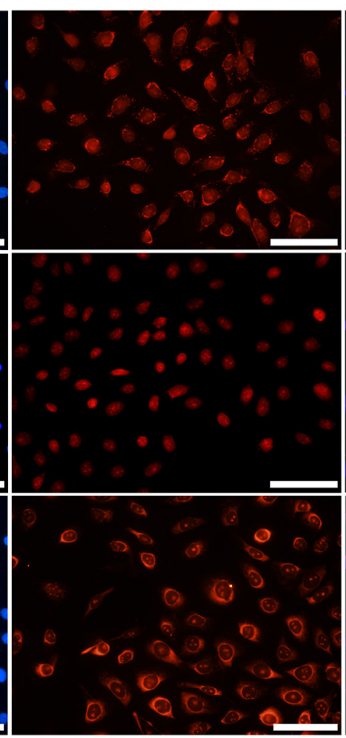

Merge
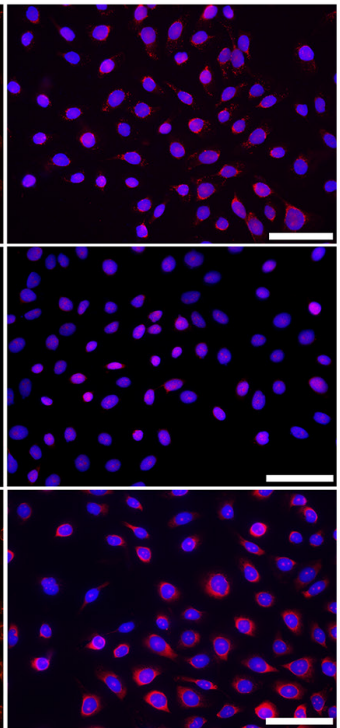

$\mathrm{F}$

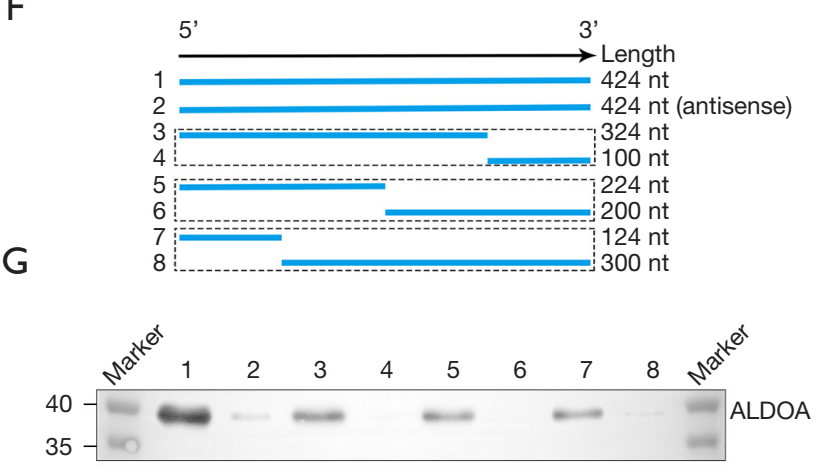

$\mathrm{H}$
B

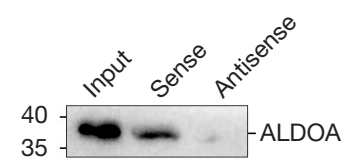

C

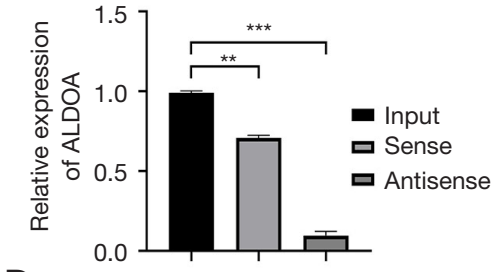

$\mathrm{D}$

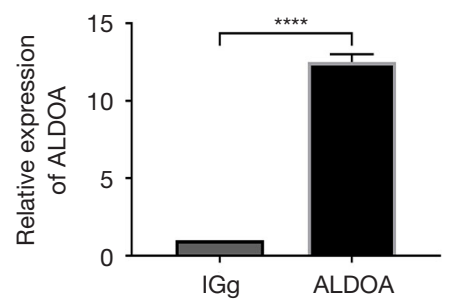

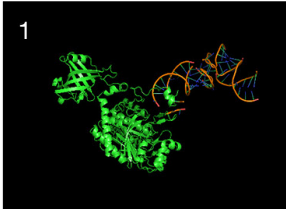

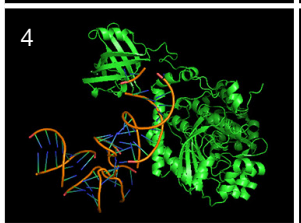

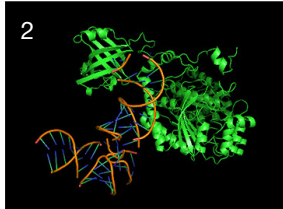

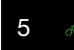

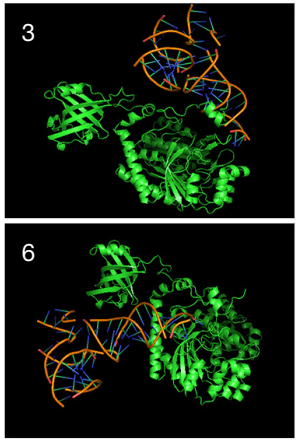
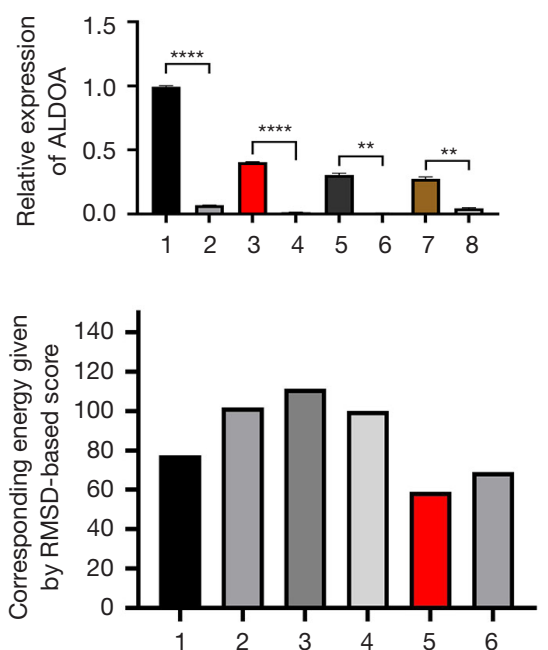

Figure 3 AC122108.1 directly interacts with ALDOA. (A) RNA-FISH images of the subcellular localization of AC122108.1 in PC14/B cells (immunofluorescence staining, scale bar: $50 \mu \mathrm{m}$ ). (B-E) Imaging of the RNA pulldown experiment followed by Western blot and silver staining. (F-H) WB analysis of ALDOA pulled down by full-length or truncated AC122108.1. (I,J) 3D structure prediction of AC122108.1 binding to ALDOA complexes $\left({ }^{* *} \mathrm{P}<0.01,{ }^{* * *} \mathrm{P}<0.001,{ }^{* * * *} \mathrm{P}<0.0001\right)$. ALDOA, aldolase $\mathrm{A}$. 
A

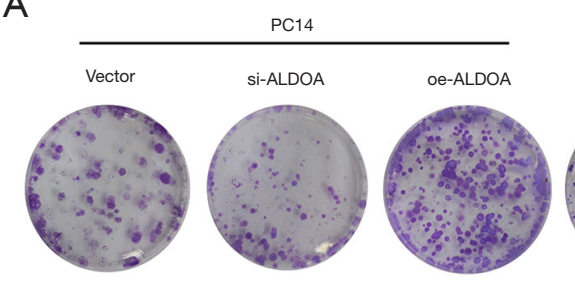

C

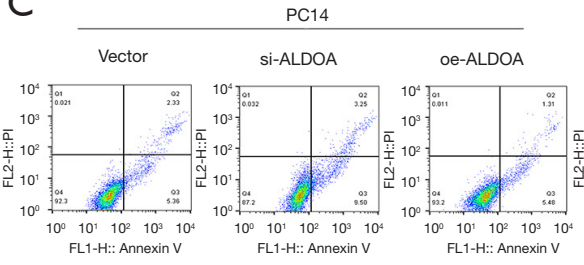

$\mathrm{E}$

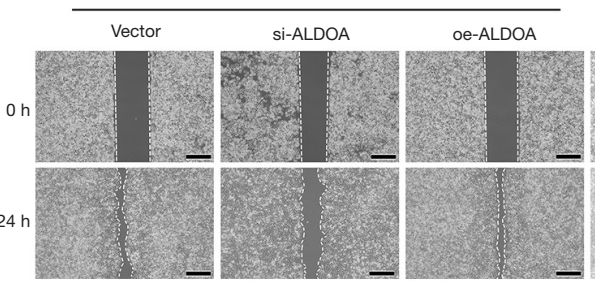

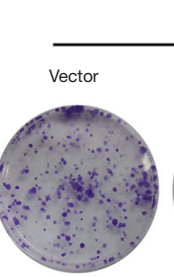

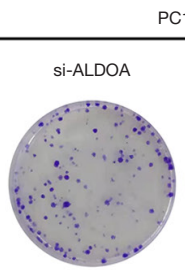

PC14B
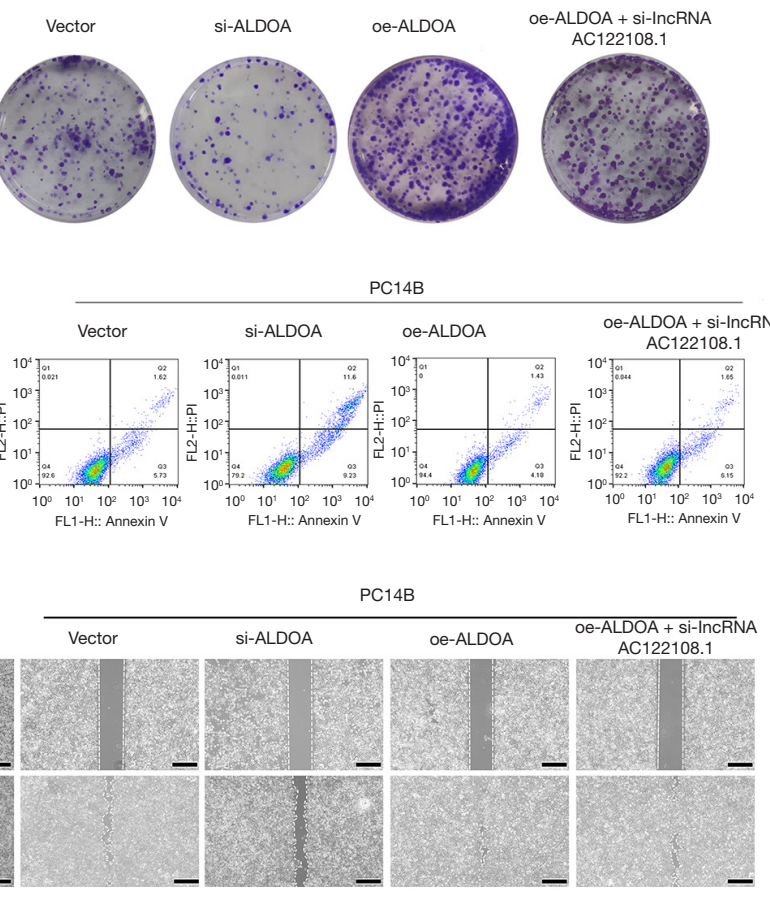

PC14B

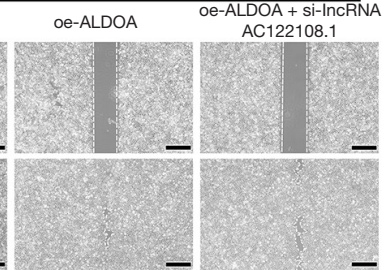

B

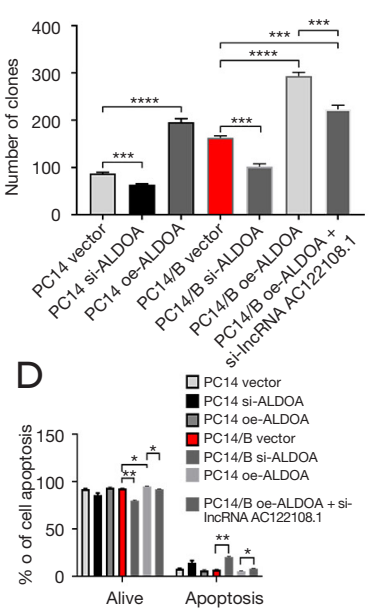

$\mathrm{F}$
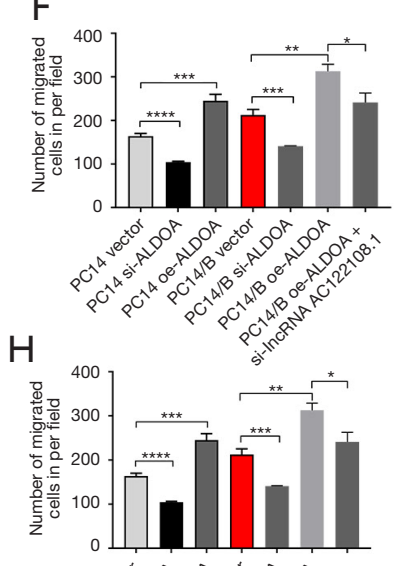

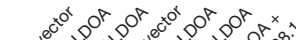
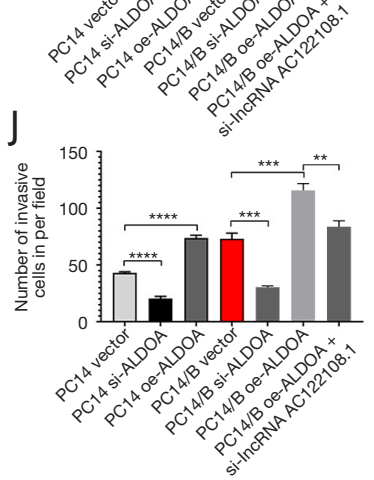

Figure 4 The effect of the low and over expression of ALDOA (si-ALDOA, oe-ALDOA) and AC122108.1 following knockdown on LAD cell proliferation, cell cycle, apoptosis, migration, and invasion. $(\mathrm{A}, \mathrm{B})$ The clone formation ability of the LAD cells transfected by siALDOA, oe-ALDOA, and si-AC122108.1 (crystal violet staining). (C,D) The cell apoptosis of LAD cells transfected by si-ALDOA, oeALDOA, and si-AC122108.1. (E,F) Wound healing assay of the LAD cells transfected by si-ALDOA, oe-ALDOA, and si-AC122108.1 (scale bar: $200 \mu \mathrm{m}$ ). (G,H) Cell migration assay of the LAD cells transfected by si-ALDOA, oe-ALDOA, and si-AC122108.1 (crystal violet staining, scale bar: $25 \mu \mathrm{m}$ ). (I,J) Cell invasion assay of the LAD cells transfected by si-ALDOA, oe-ALDOA and si-AC122108.1 (crystal violet staining, scale bar: $\left.25 \mu \mathrm{m} ;{ }^{*} \mathrm{P}<0.05,{ }^{* *} \mathrm{P}<0.01,{ }^{* * *} \mathrm{P}<0.001,{ }^{* * * *} \mathrm{P}<0.0001\right)$. ALDOA, aldolase $\mathrm{A} ; \mathrm{LAD}$, lung adenocarcinoma. 
A functional RNA-protein complex composed of AC122108.1 and ALDOA played a role in the WNT/ $\beta$-Catenin signaling patbway

The seahorse assay was used to test the extracellular acidification rate of different groups of LAD cells. Compared to the other groups, no change in glycolysis, the glycolytic capacity and the glycolytic reserve occurred in PC14/B cells transfected with si-AC122108.1 (Figure 5A-5D). Thus, we hypothesized that binding AC122108.1 to ALDOA affects LAD cells through the nonenzymatic pathway.

As aldolase proteins are Wnt signaling regulators, ALDOA could activate Wnt signaling by a GSK-3 $\beta$ dependent mechanism and positively regulate the canonical Wnt pathway through the non-enzymatic pathway. To further explore whether the RNA-protein complex composed of AC122108.1 and ALDOA plays a similar role in the canonical Wnt signaling pathway, we performed coimmunoprecipitation (co-IP) experiments. The results showed that ALDOA and GSK-3 $\beta$ interacted with each other reciprocally, and that the knockdown of AC122108.1 attenuated the interaction of ALDOA and GSK-3 $\beta$. Notably, the inhibitory effect of ALDOA knockdown was more obvious in LAD cell lines compared to control cell lines (Figure 5E, 5F).

After AC122108.1 was knocked down in PC14/B cells, the total amount of $p-\beta$-catenin increased significantly, while the total amount of $\beta$-catenin decreased slightly (Figure $5 G, 5 H$ ). The results were similar to those on the knockdown of ALDOA in LAD cell lines (Figure 5I,57), the only difference was that AC122108.1 did not suppress the expression of ALDOA. Overall, we speculated that AC122108.1 may be involved in the process of $\beta$-catenin degradation by forming an RNA-protein complex with ALDOA and inhibiting the $\beta$-catenin degradation complex by the same mechanism as that of ALDOA. The knockdown of both AC122108.1 and ALDOA promoted the degradation of $\beta$-catenin, and further downregulated $\beta$-catenin nuclear translocation and the expression of several downstream target genes that are indirectly involved in various pathological and tumorigenic processes in $\mathrm{LAD}$ cell lines (Figure $5 K-5 N$ ).

For completeness, the overexpression of ALDOA was examined to further verify our hypothesis above (Figure 5O-5R). Together, the results supported our prediction that AC122108.1 acts to activate the WNT/ $\beta$-Catenin signaling pathway by a functional RNA-protein complex composed of AC122108.1 and ALDOA. The above epigenetic experiments of the LAD cell lines were also further verified.

\section{AC122108.1 promoted LAD cell metastasis in vivo}

To evaluate the effects of AC122108.1 on in situ tumor formation and BM, PC14/B cells with AC122108.1 knockdown were injected into nude mice through the internal carotid artery. Both tumor volume and the number of cancer foci were significantly more decreased in the AC122108.1-knockdown group than the control group, and the HE staining analysis indicated that the AC122108.1knockdown group had better OS after 6 weeks than the control group' (Figure 6A-6C). As diagnostic markers of LAD, the expression of Napsin A and cytokeratin 7 (CK7) in brain tissues indicated that the pathological type of the two groups was LAD, and the decreased Napsin A expression levels implied that the control group had more poorly differentiated LAD and a poor prognosis (24) (Figure 6D). Finally, the immunofluorescence staining of the metastatic tumors showed that inhibiting the expression of AC122108.1 significantly reduced the expression of $\beta$-catenin, and more importantly, the translocation of ALDOA and $\beta$-catenin from the cytoplasm to the nucleus was obviously enhanced (Figure $6 E$ ). These findings showed that the knockdown of the expression of AC122108.1 in vivo significantly impaired LAD tumorigenicity.

\section{AC122108.1 was clinically associated with the expression of ALDOA and $\beta$-Catenin in LAD patients}

To further investigate whether the above findings were clinically relevant, the expression of AC122108.1 in the LAD clinicopathological tissues and the correlation between ALDOA and $\beta$-catenin expression were examined. The results clearly showed that the expression of ALDOA and $\beta$-catenin was increased in the LAD clinicopathological tissue, and the translocation from the cytoplasm to the nucleus was increased in the lung tissue group, which had a high expression of AC122108.1 (Figure 7). However, the expression of AC122108.1 had little effect on ALDOA expression in the brain tissue group, and the translocation of ALDOA from the cytoplasm to the nucleus became more pronounced. In relation to $\beta$-catenin in the brain tissue group, the results were similar to those of previous studies of the lung tissue group. These findings suggest that AC122108.1 increased the expression of nuclear $\beta$-catenin 
A

Glycolytic function

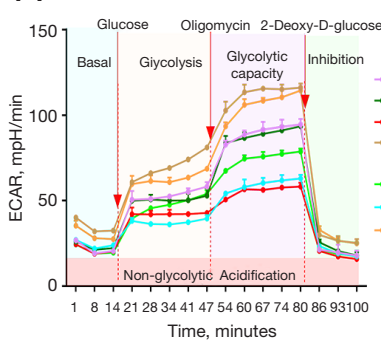

Time, minutes

$\mathrm{E}$
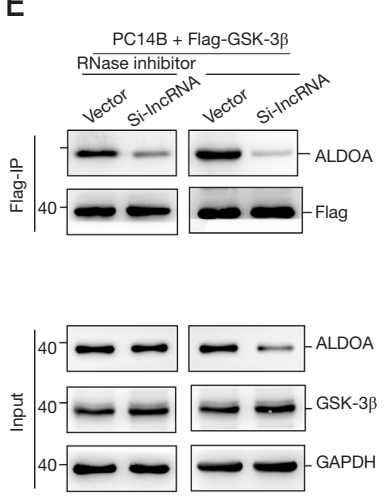

K

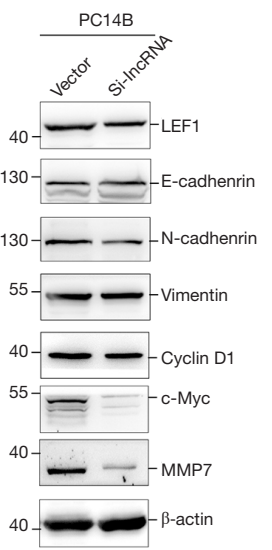

$\mathrm{F}$
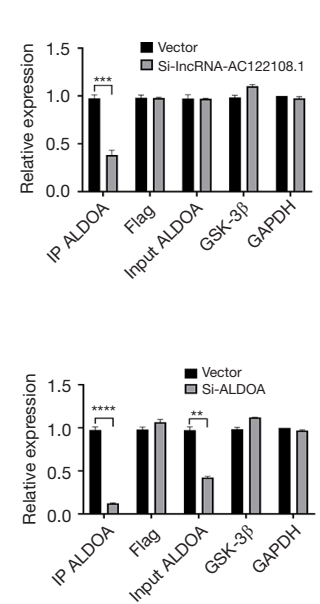

B

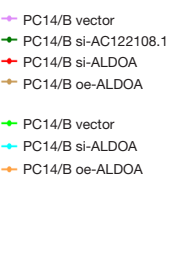

$\rightarrow$ PC14/B vector
$\rightarrow$ PC14/B si-AC122108.1
$\rightarrow$ PC14/B si-ALDOA

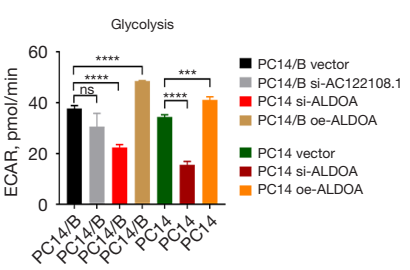

G

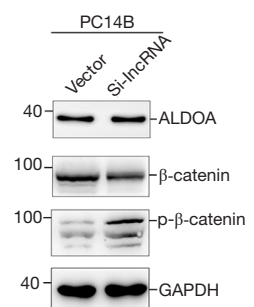

$\mathrm{H}$

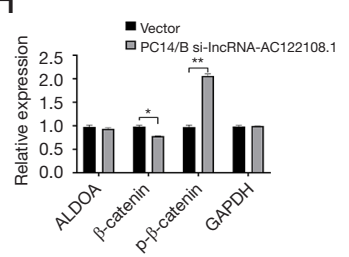

M

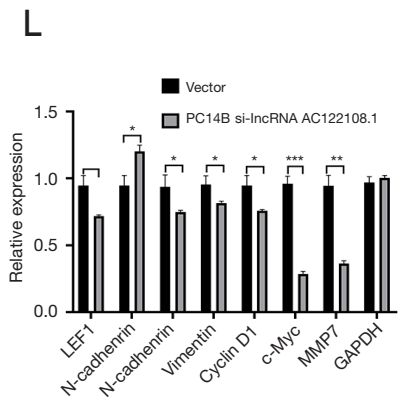

C
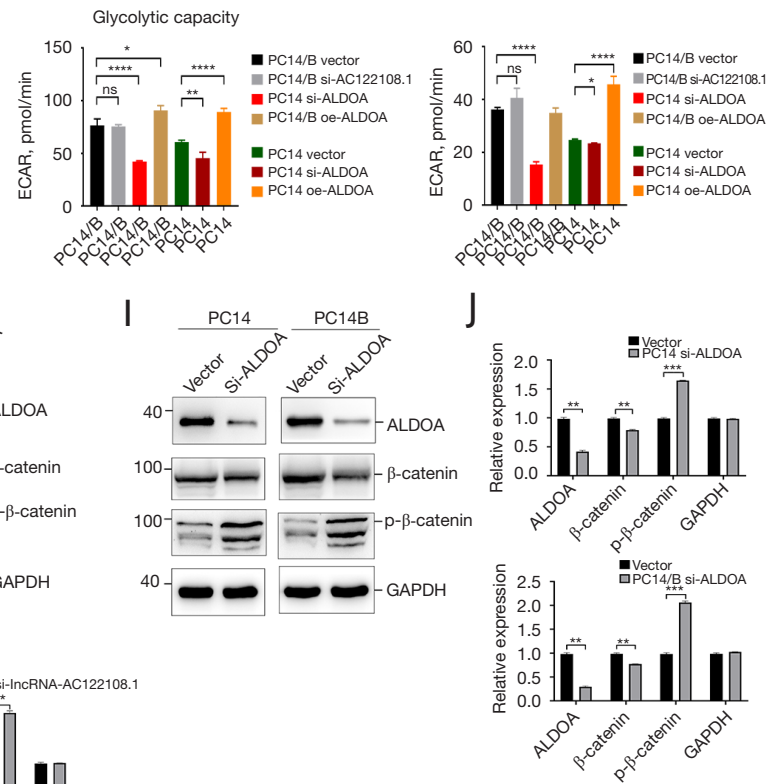

$\mathrm{N}$
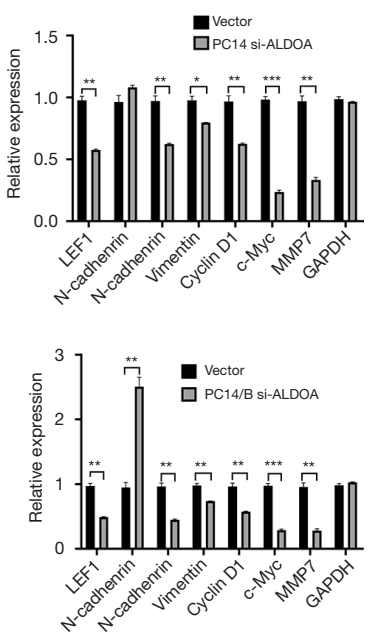

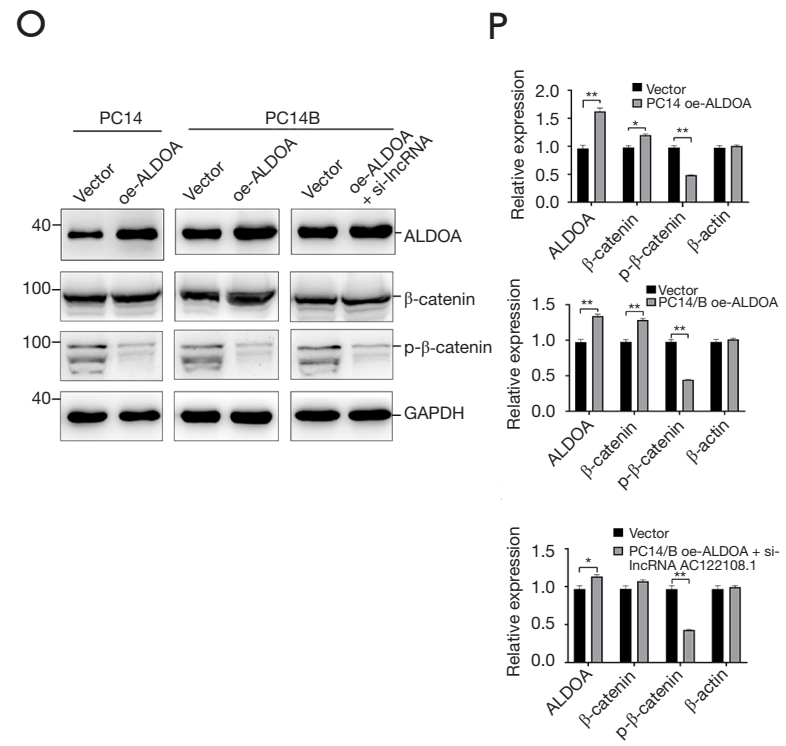

R
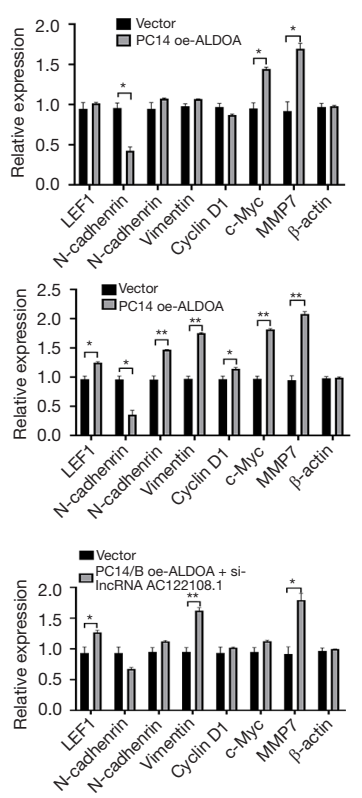

Figure 5 A functional RNA-protein complex composed of AC122108.1 and ALDOA plays a role in the WNT/ $\beta$-Catenin signaling pathway. (A-D) The extracellular acidification rate of the LAD cells transfected by si-ALDOA and si-AC122108.1. (E,F) GSK-3 $\beta$ interacts with ALDOA proteins by co-immunoprecipitation with anti-FLAG M2 beads. (G-J) The differential expression of $\beta$-catenin and p- $\beta$-catenin in LAD cells transfected by si-ALDOA and si-AC122108.1. (K-N) The differential expression of EMT (pithelial-mesenchymaltransition)related proteins and downstream-target proteins of the $\mathrm{WNT} / \beta$-Catenin signaling pathway in LAD cells transfected by si-ALDOA and siAC122108.1. (O,P) The differential expression of $\beta$-catenin and $\mathrm{p}-\beta$-catenin in LAD cells transfected by oe-ALDOA and si-AC122108.1. $(\mathrm{Q}, \mathrm{R})$ The differential expression of EMT-related proteins and downstream target proteins of the WNT/ $\beta$-Catenin signaling pathway in LAD cells transfected by oe-ALDOA and si-AC122108.1 $\left({ }^{*} \mathrm{P}<0.05,{ }^{* *} \mathrm{P}<0.01,{ }^{* * *} \mathrm{P}<0.001,{ }^{* * *} \mathrm{P}<0.0001\right)$. ALDOA, aldolase A; LAD, lung adenocarcinoma; EMT, epithelial-mesenchymal transition.

in LAD patients, and clinically contributed to the activation of the WNT/ $\beta$-catenin signaling pathway (Figure 8).

\section{Discussion}

LAD is a main pathological subtype of lung cancer and is one of the most aggressive malignant cancers worldwide (25-27). Despite advancements in its clinical diagnosis and treatment, the overall prognosis of most LAD patients remains poor (28). BM is a common complication of LAD, and its incidence in advanced LAD is as high as $30-50 \%$ (29). BM can significantly reduce the survival and quality of life of LAD patients, and the molecular mechanisms of BM in LAD have attracted increasing interest from researchers seeking to identify early diagnostic markers and develop targeted treatments. Previous studies have shown that lncRNAs participate in the progression of human cancers, and can serve as biomarkers and therapeutic targets for tumors (30). As hallmarks of LAD, migration, invasion, and metastasis are regulated by lncRNAs $(31,32)$, and many lncRNAs also play a critical role in LAD BM $(33,34)$. Initially, lncRNA was considered to be RNA without biological functions. However, subsequent studies have shown that lncRNA plays an important role in transcription, post-transcriptional and epigenetic processes. LncRNA has a structure similar to mRNA, with a promoter structure and a PolyA tail. It has been confirmed that lncRNA plays an important regulatory role in cell proliferation, differentiation, development, apoptosis and metastasis. Studies have shown that lncRNA LET can inhibit the proliferation and EMT of lung adenocarcinoma cells (35,36). ELK1-induced lncRNA HOXA10AS upregulation promotes the progression of lung adenocarcinoma by increasing $W n t / \beta$-catenin signaling (37). However, currently, very few specific lncRNAs have been confirmed to be closely associated with LAD BM, and the underlying mechanism of action remains unclear.

First, transcriptome analyses were carried out on the 
A

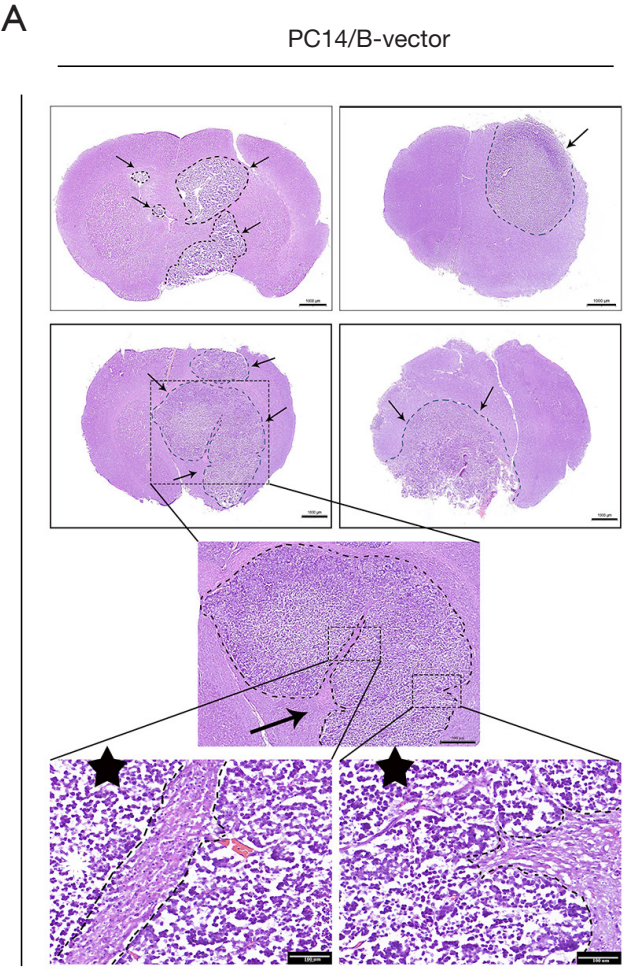

B
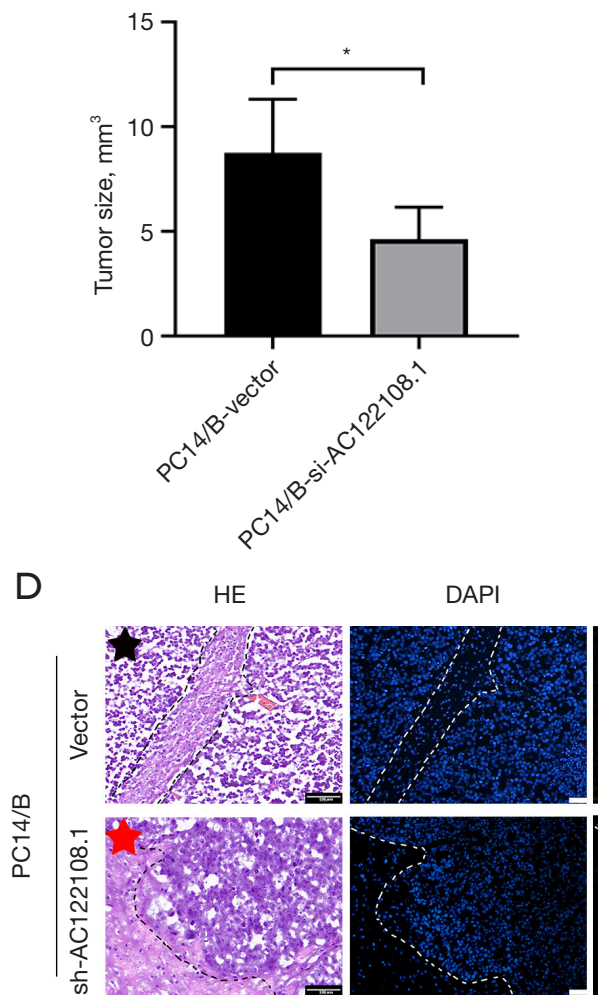

C
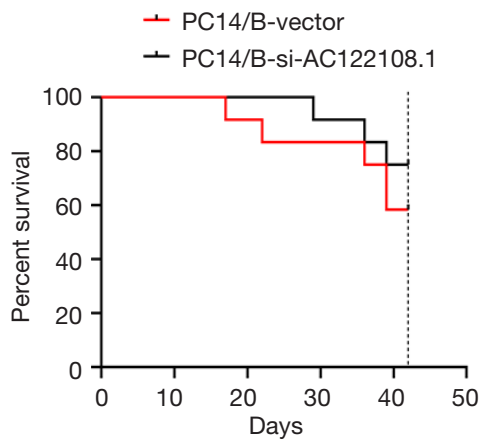

Napsin A

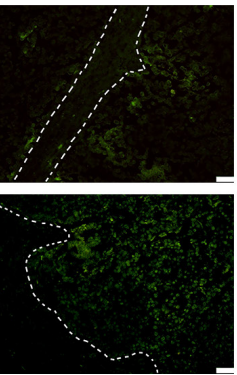

- PC14/B-vector

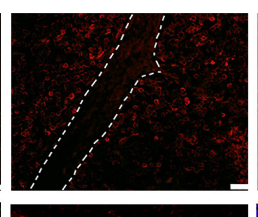

PC14/B-sh-AC122108.1
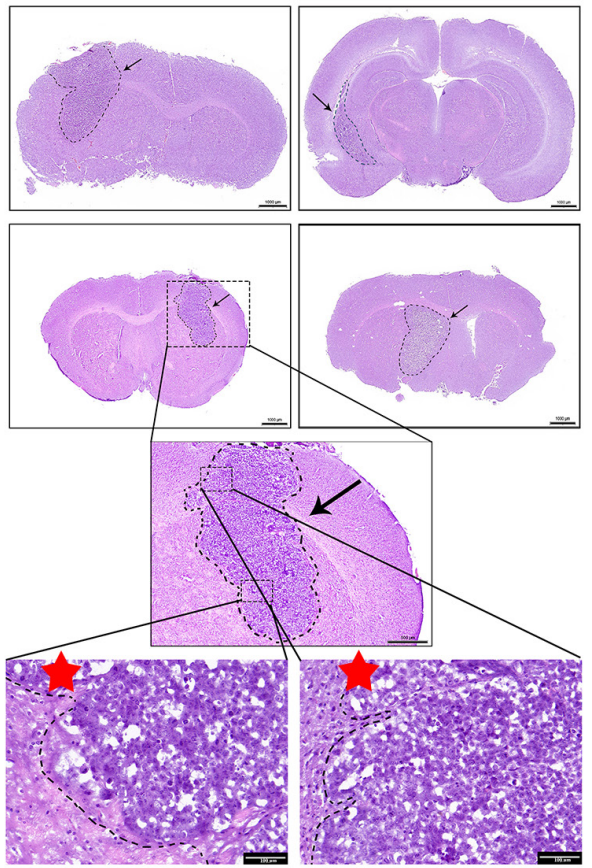

Merge

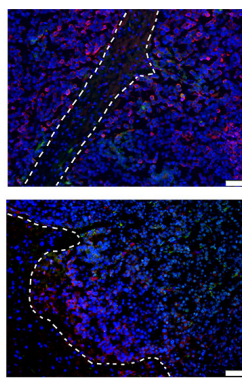


E

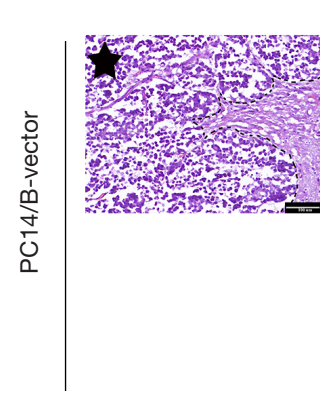

DAPI
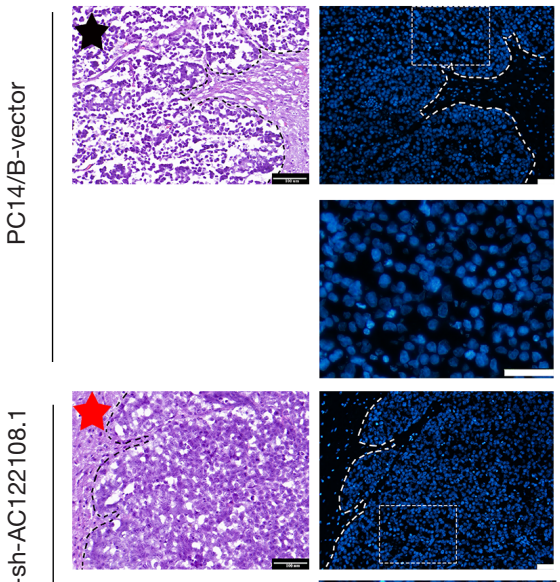

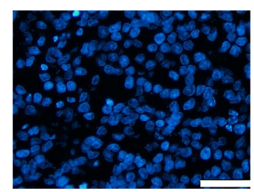

ALDOA
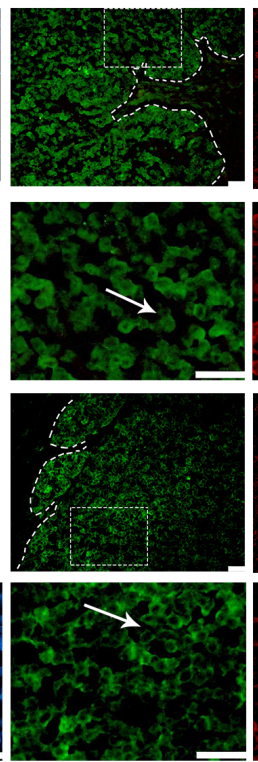

$\beta$-catenin
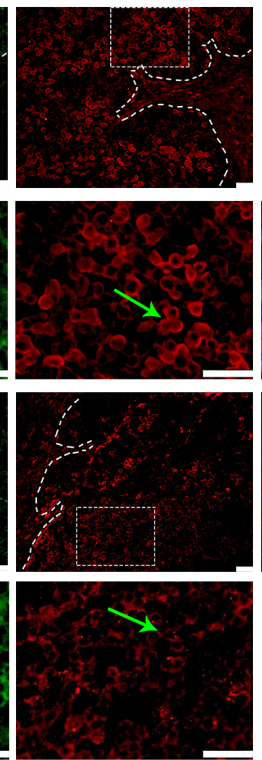

Merge
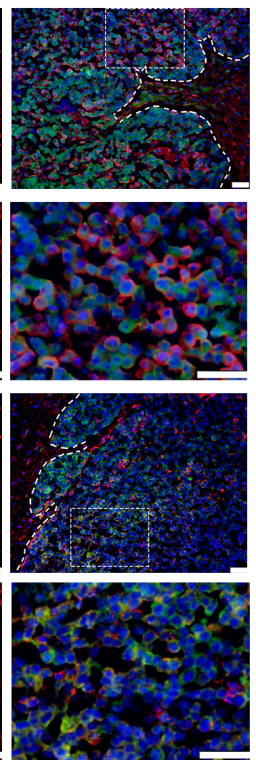

Figure 6 AC122108.1 promotes LAD cell metastasis in vivo. (A-C) The HE staining and image analysis showed the metastatic lesions in different groups of mice brains (hematoxylin and eosin staining, scale bar: 1,000 $\mu \mathrm{m}, 500 \mu \mathrm{m}, 100 \mu \mathrm{m}$; the black arrows indicate metastatic nodules, $\left.{ }^{*} \mathrm{P}<0.05\right)$. (D) Expression of Napsin A and CK7 as shown by immunofluorescence in different groups (black scale bar: 100 4 , white scale bar: $50 \mu \mathrm{m}$; the black star indicates injectable cells transfected by a vector; the red star indicates injectable cells transfected by shAC122108.1). (E) Expression of ALDOA and $\beta$-catenin shown by immunofluorescence in different groups (black scale bar: $100 \mu$ m, white scale bar: $50 \mu \mathrm{m}$; the black star indicates injectable cells transfected by a vector; the red star indicates injectable cells transfected by shAC122108.1; the white arrows indicate ALDOA nuclear translocation; the green arrows indicate $\beta$-catenin nuclear translocation). ALDOA, aldolase A; LAD, lung adenocarcinoma.

$\mathrm{BM}$ cell line (PC14/B) and the control cell line (PC14) to determine the differentially expressed genes and the quality of the transcriptome data. In our previous results, we identified a new lncRNA (i.e., AC122108.1), which was significantly upregulated in the BM cell line and the BM tumor tissue of LAD patients. In the present study, the malignant behaviors associated with BM (i.e., proliferation, migration, invasion, and metastasis) were obviously inhibited by the loss of AC122108.1. Thus, AC122108.1 appears to act as a cancer promoting factor in $\mathrm{BM}$ tumorigenesis, and other malignant behaviors of LAD.

LncRNAs are a large and diverse group of transcripts with different genomic origin, subcellular localization, and functional pathways that affect gene expression at epigenetic, transcriptional and post-transcriptional levels through a variety of mechanisms (38). However, the way in which the specific localization of IncRNAs mediates their functions and the exact rules lncRNAs follow in gene regulation remain largely unknown (39). As a completely unknown lncRNA, AC122108.1 might exert its functions in multiple ways. We were particularly interested in lncRNAprotein interactions. Consequently, a series of experiments, such as FISH, pulldown, a MS analysis, RIP, PAGE silver staining, and qRT-PCR were used to identify the biological mechanisms of AC122108.1 (40).

As our previous results showed, ALDOA stood out among all proteins that were predicted to interact with AC122108.1, and is not only a key enzyme in glycolysis, but also an independent clinical prognostic marker of human cancers $(41,42)$. There is increasing evidence that ALDOA performs functions in many human malignancies, such as pancreatic cancer $(43,44)$, colorectal cancer $(45)$, colon cancer (46), bile duct cancer (47), bladder cancer (48), gastric cancer (49), and lung cancer $(15,16,50-53)$. As a key enzyme, ALDOA accelerates glycolysis to promote the migration and invasion of lung cancer (53). Due to its non-enzymatic function, ALDOA might affect the cellcycle progression of NSCLC in a manner independent of glycolysis (15). ALDOA promotes lung cancer invasion, migration, and metastasis by activating the HIF-1 $\alpha$ 
A

\begin{tabular}{c} 
A \\
A \\
\\
0 \\
0 \\
0 \\
\hline 0 \\
0 \\
\hline \\
\hline
\end{tabular}

B
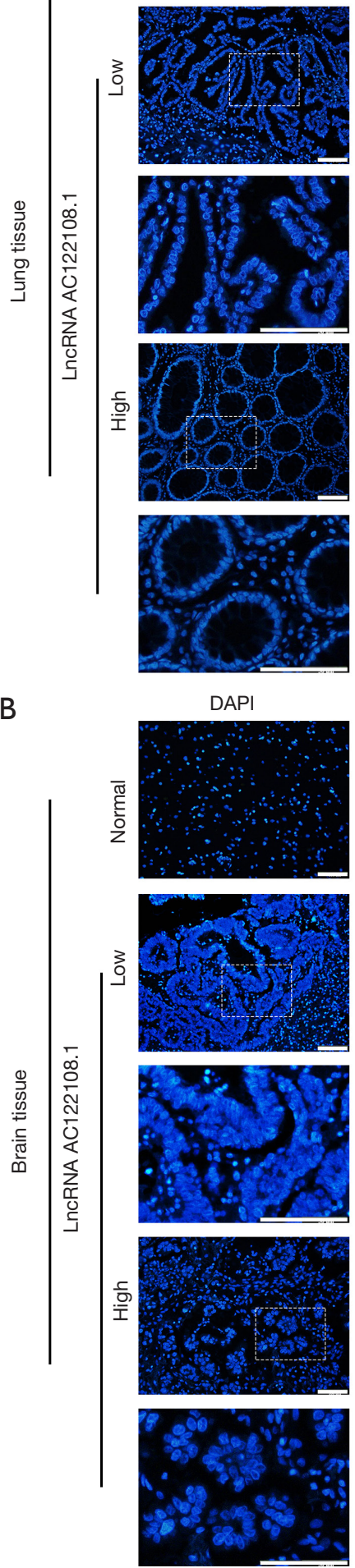

艺

들

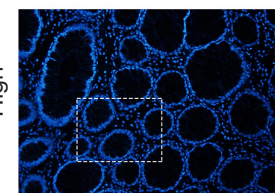

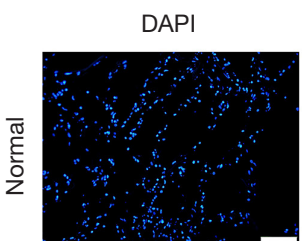

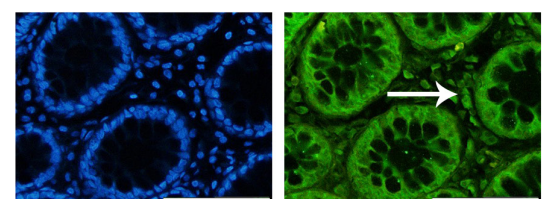

ALDOA
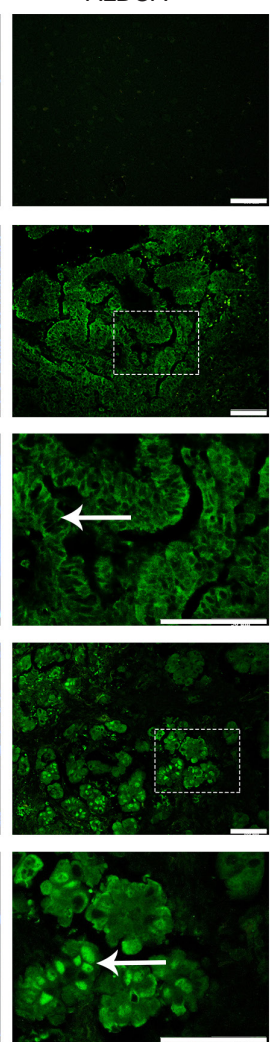

$\beta$-catenin
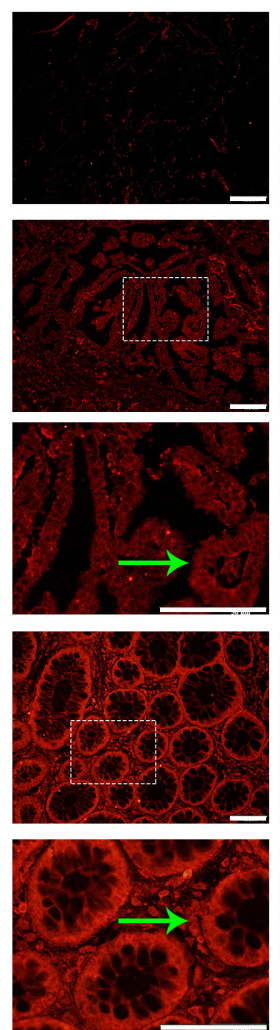

$\beta$-catenin
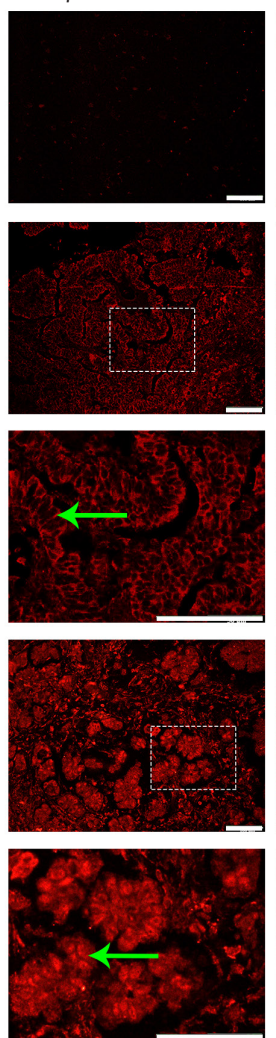
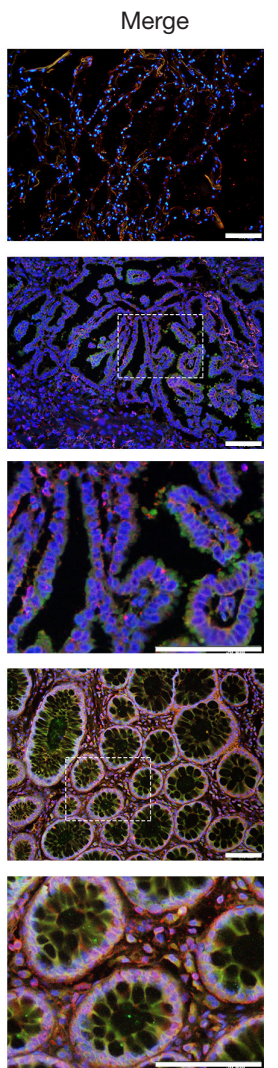

Merge
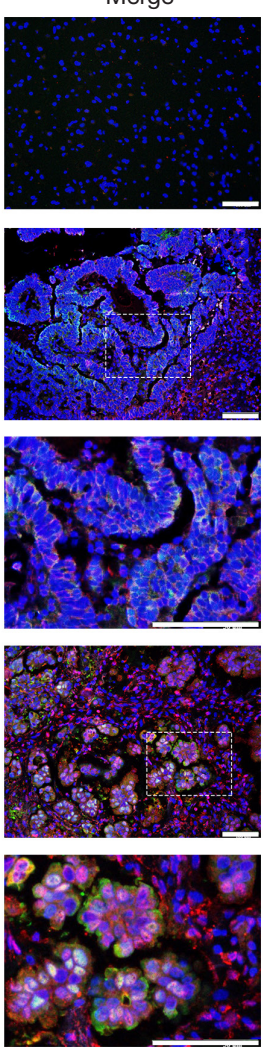

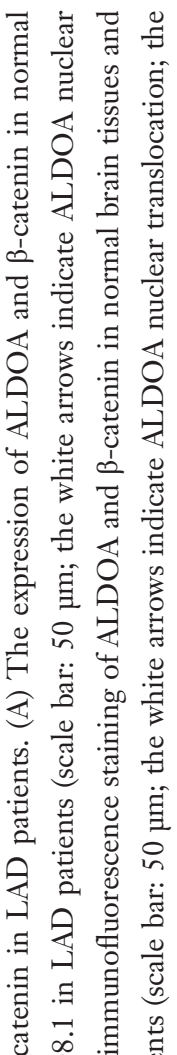

¿ च ָ

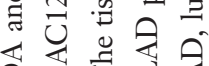

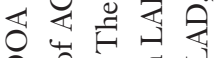
卷争

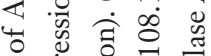
ป 응 근

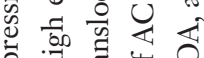
층

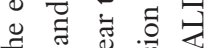
I 글 节矛苛 . 3 है

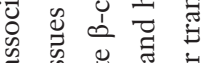

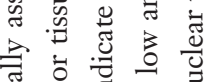

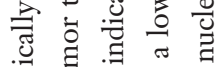
手吉点言 क ष्ठ 3 है क वे $\vec{\infty}$ bo 忩吉 吉声塄

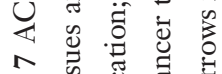
\% .

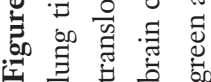




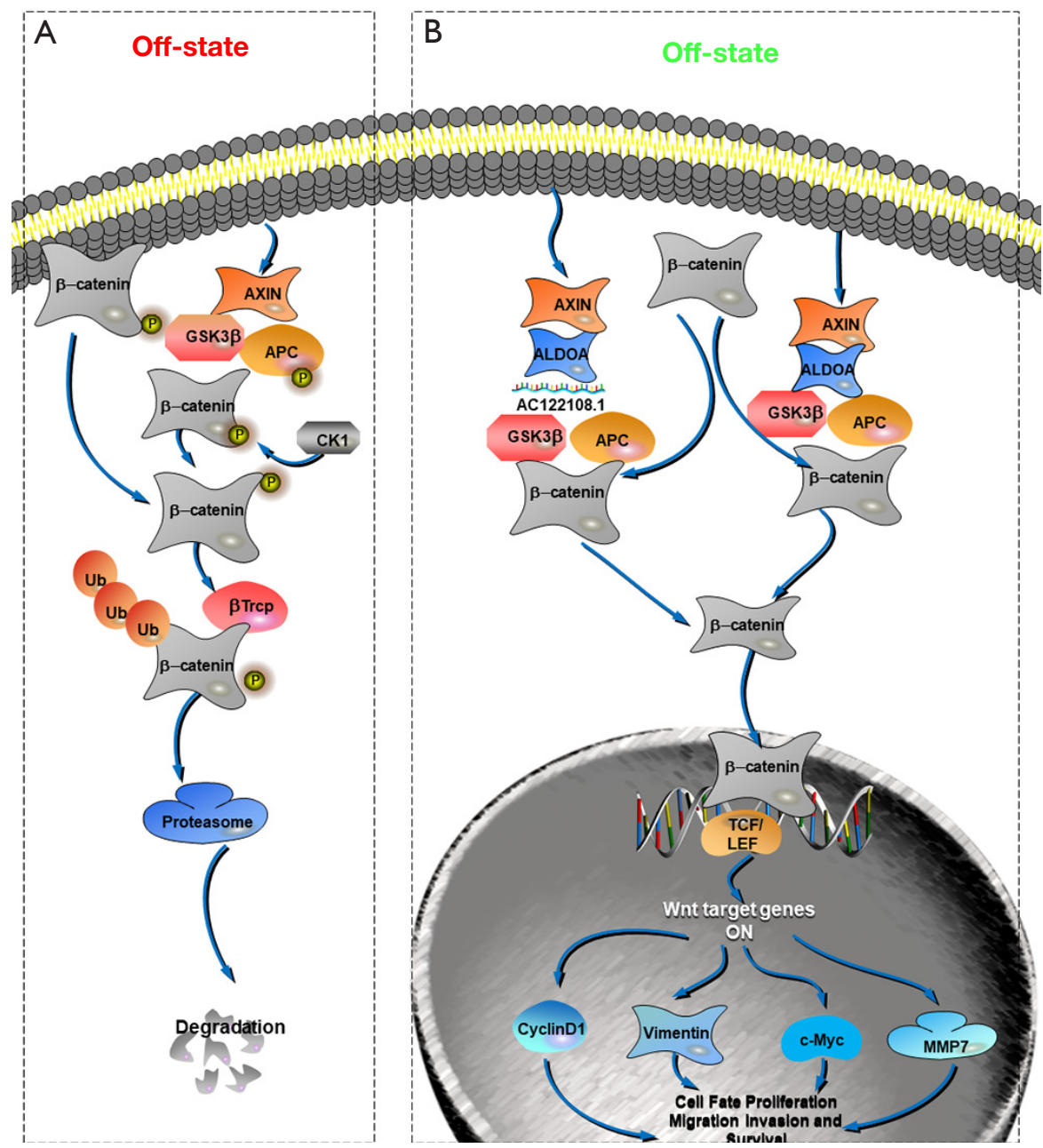

Figure 8 A hypothetic model of the role of the AC122108.1 in Wnt signaling stimulation. (A) Wnt signaling is off-state; $\beta$-catenin is degraded by a dedicated cytoplasmic " $\beta$-catenin degradation protein complex". (B) Wnt signaling is on-state in PC14/B cells that express high levels of the AC122108.1; a complex of AC122108.1 and ALDOA binds to GSK-3 $\beta$, disassembling the " $\beta$-catenin degradation protein complex". The activation of the Wnt cascade leads to the accumulation and nuclear translocation of $\beta$-catenin, and results in the expression of Wnt target genes. ALDOA, aldolase A.

(hypoxia inducible factor 1 subunit alpha)/MMP9 (matrix metallopeptidase 9) axis (16). ALDOA plays an important role in inducing cancer stemness by inhibiting miR-145 expression and activating octamer-binding protein 4 (Oct4) transcription (50). Unfortunately, the mechanism of action of ALDOA in cancer remains unknown. To elucidate how AC122108.1 is involved in the BM of LAD development, we analyzed the correlation of AC122108.1 expression with clinicopathological factors. We found a significant correlation between AC122108.1 expression and tumor $(\mathrm{T})$, node $(\mathrm{N})$, and metastasis $(\mathrm{M})$ staging and expressions of Ki-67. These results provided further evidence that
AC122108.1 could be used as a potential biomarker to evaluate the prognosis of $\mathrm{LAD}$ patients.

In this study, we confirmed that AC122108.1 had no significant effect on the metabolic enzyme activity or expression of ALDOA. However, we found that AC122108.1 and ALDOA were very similar in terms of their primary location in the cytoplasm, and the behaviors of AC122108.1-and ALDOA-knockdown PC14/B cells were also similar. In addition, the knockdown of AC122108.1 reduced the tumorigenicity of ALDOA as demonstrated by co-transfections of si-AC122108.1 and oe-ALDOA. For these reasons, we believe that there is a certain collaborative 
functional relationship between AC122108.1 and ALDOA. Additionally, the application of segmented pulldown and lncRNA-protein structure prediction (50) confirmed that they may form a structural complex (54). Thus, we hypothesized that they can be integrated into RNA-protein structural functional complexes that act as cancer promoting factors.

The GO and KEGG pathway enrichment analyses strongly indicated that AC122108.1 regulates tumor progression by the $\mathrm{Wnt} / \beta$-catenin signaling pathway, which is closely related to the invasion of LAD (55) (Figure S1). Additionally, it has been proven that aldolase can regulate the stability of the $\beta$-catenin destruction complex to activate the $\mathrm{Wnt} / \beta$-catenin signaling pathway in colorectal cancer (17). Based on the co-IP results on ALDOA and GSK-3 $\beta$, and the results on the expression of $\beta$-catenin and $\mathrm{p}-\beta$-catenin, we believe that $\mathrm{AC} 122108.1$ binds to ALDOA, which in turn activates the Wnt signaling pathway and affects the $\beta$-catenin destruction complex (56). However, the mechanism by which the Wnt signaling cascade is triggered is not fully understood. We also conducted immunofluorescence and Western blot assays to confirm the accumulation and nuclear translocation of $\beta$-catenin (57) resulting in the expression of $\mathrm{Wnt}$ downstream genes, such as vimentin, cyclinD1, c-Myc, and MMP7. Overall, we firmly believe that the binding of AC122108.1 to ALDOA promotes BM in LAD via the $\mathrm{Wnt} / \beta$-catenin signaling pathway.

Our study had several limitations. LncRNAs participate in chromatin condensation, nuclear body formation, and function. By acting as molecular decoys, LncRNAs are involved in mRNA splicing, translational inhibition, and miRNA sponging and compete for miRNA binding sites on mRNA $(38,39)$. We only studied the effect of AC122108.1 on the regulation of $\beta$-catenin degradation in LAD through ALDOA; thus, we do not know the specific mechanism by which AC122108.1 interacts ALDOA. Recent research on RBP has shown that LncRNAs are involved in protein modification, including ubiquitination and phosphorylation; for example, long non-coding RNA, dendritic cell differentiation (lnc-DC) promotes STAT3 phosphorylation by binding to STAT3 directly in the cytoplasm to regulate human dendritic cell differentiation (58). NF- $\kappa \mathrm{B}$ interacting lncRNA directly blocks I $\kappa$ phosphorylation and suppresses breast cancer metastasis (59). LincRNA-p21mediates HIF$1 \alpha$ ubiquitination by disrupting the VHL (Von HippelLindau tumor suppressor)-HIF-1 $\alpha$ interaction to promote glycolysis under hypoxia (60). In addition, the latest research shows that ALDOA-S36 phosphorylation has a strong tumor promoting effect by inducing a mutation of catenin beta 1(CTNNB1) (61). Based on these findings, we conducted further research and verified that AC122108.1 does not phosphorylate, ubiquitinylate, or acetylate ALDOA. Currently, it has only been established that there is an interaction between AC122108.1 and ALDOA, and the specific mechanism by which this occurs require furthers exploration. Further, in our immunofluorescence experiments, we found that the high expression of AC122108.1 was accompanied by the accumulation of ALDOA in the nucleus in both mice and human tissues. The question of whether AC122108.1 promotes the nuclear transfer of ALDOA and induces LAD progression needs to be verified in the future.

In summary, the expression of specific lncRNAs has been reported to be correlated with clinical features in LAD, and the utility of lncRNAs in the diagnosis and prognosis of LAD has significant clinical value $(33,34,55)$. In this study, we demonstrated that AC122108.1 was significantly upregulated in LAD BM cells and tissues, and that the expression of AC122108 was correlated with the malignancy of tumors, including tumor proliferation, apoptosis, invasion, migration, and metastasis. The expression level of AC122108.1 can be used to predict the prognosis of patients with $\mathrm{BM}$ and $\mathrm{LAD}$ to a certain extent. Blocking the production of AC122108.1 may be an effective strategy for cancer treatment. To a certain extent, the expression level of AC122108.1 can be used to predict BM and the prognosis of LAD patients. Additionally, both the in vivo and in vitro studies showed that a complex composed of AC122108.1 and ALDOA inhibited the destruction of $\beta$-catenin, resulting in the expression of Wnt target genes. Consequently, the binding of AC122108.1 to ALDOA leads to the " $\beta$-catenin destruction complex" inactivation and accumulation and the nuclear translocation of $\beta$-catenin, and promotes LAD $\mathrm{BM}$ and progression through the $\mathrm{Wnt} / \beta$-catenin signaling pathway. Thus, the AC122108.1/ALDOA/ $\beta$-catenin axis might act as a new post-transcriptional regulatory mechanism and serve as a potential therapeutic target for $\mathrm{LAD} \mathrm{BM}$ diagnosis and treatment.

\section{Acknowledgments}

Funding: This work was supported by Jinan Science and Technology Plan (grant No. 201907049), China National Natural Science Foundation (grant No. 81771270), Shandong Provincial Natural Science Foundation (grant 
No. 202019088), the Natural Science Foundation of Shandong Province (grant No. ZR2014HM010) and Shandong Province Science and Technology Development Program (grant No. 2015GSF118164).

\section{Footnote}

Reporting Checklist: The authors have completed the ARRIVE reporting checklist. Available at https://dx.doi. org/10.21037/atm-21-5707

Data Sharing Statement: Available at https://dx.doi. org/10.21037/atm-21-5707

Conflicts of Interest: All authors have completed the ICMJE uniform disclosure form (available at https://dx.doi. org/10.21037/atm-21-5707). The authors have no conflicts of interest to declare.

Ethical Statement: The authors are accountable for all aspects of the work in ensuring that questions related to the accuracy or integrity of any part of the work are appropriately investigated and resolved. All procedures performed in this study involving human participants were in accordance with the Declaration of Helsinki (as revised in 2013). This study was approved by the Ethics Committee of the Shandong Provincial Hospital Affiliated to Shandong University (SWYX: No. 2019-168). Written consent was obtained from all patients or their families. All animal experiments were approved under a project license (No. 2019144) granted by the Institutional Animal Care and Use Committee of Shandong University and conducted in strict accordance with its recommendations and ethical guidelines for the care and use of animals.

Open Access Statement: This is an Open Access article distributed in accordance with the Creative Commons Attribution-NonCommercial-NoDerivs 4.0 International License (CC BY-NC-ND 4.0), which permits the noncommercial replication and distribution of the article with the strict proviso that no changes or edits are made and the original work is properly cited (including links to both the formal publication through the relevant DOI and the license). See: https://creativecommons.org/licenses/by-nc-nd/4.0/.

\section{References}

1. Wang G, Xu J, Qi Y, et al. Distribution Of Brain
Metastasis From Lung Cancer. Cancer Manag Res 2019;11:9331-8.

2. Imielinski M, Berger AH, Hammerman PS, et al. Mapping the hallmarks of lung adenocarcinoma with massively parallel sequencing. Cell 2012;150:1107-20.

3. Travis WD, Brambilla E, Nicholson AG, et al. The 2015 World Health Organization Classification of Lung Tumors: Impact of Genetic, Clinical and Radiologic Advances Since the 2004 Classification. J Thorac Oncol 2015;10:1243-60.

4. Churilla TM, Chowdhury IH, Handorf E, et al. Comparison of Local Control of Brain Metastases With Stereotactic Radiosurgery vs Surgical Resection: A Secondary Analysis of a Randomized Clinical Trial. JAMA Oncol 2019;5:243-7.

5. van Meerbeeck JP, Fennell DA, De Ruysscher DK. Smallcell lung cancer. Lancet 2011;378:1741-55.

6. Tsao MN, Xu W, Wong RK, et al. Whole brain radiotherapy for the treatment of newly diagnosed multiple brain metastases. Cochrane Database Syst Rev 2018;1:CD003869.

7. Zhou J, Xiao H, Yang X, et al. Long noncoding RNA CASC9.5 promotes the proliferation and metastasis of lung adenocarcinoma. Sci Rep 2018;8:37.

8. Sarropoulos I, Marin R, Cardoso-Moreira M, et al. Developmental dynamics of lncRNAs across mammalian organs and species. Nature 2019;571:510-4.

9. Xiong XD, Ren X, Cai MY, et al. Long non-coding RNAs: An emerging powerhouse in the battle between life and death of tumor cells. Drug Resist Updat 2016;26:28-42.

10. Atianand MK, Caffrey DR, Fitzgerald KA. Immunobiology of Long Noncoding RNAs. Annu Rev Immunol 2017;35:177-98.

11. Schmitt AM, Chang HY. Long Noncoding RNAs in Cancer Pathways. Cancer Cell 2016;29:452-63.

12. Shen L, Chen L, Wang Y, et al. Long noncoding RNA MALAT1 promotes brain metastasis by inducing epithelial-mesenchymal transition in lung cancer. J Neurooncol 2015;121:101-8.

13. Nakagawa T, Endo H, Yokoyama M, et al. Large noncoding RNA HOTAIR enhances aggressive biological behavior and is associated with short disease-free survival in human non-small cell lung cancer. Biochem Biophys Res Commun 2013;436:319-24.

14. Wucher V, Legeai F, Hédan B, et al. FEELnc: a tool for long non-coding RNA annotation and its application to the dog transcriptome. Nucleic Acids Res 2017;45:e57.

15. Zhang F, Lin JD, Zuo XY, et al. Elevated transcriptional 
levels of aldolase A (ALDOA) associates with cell cyclerelated genes in patients with NSCLC and several solid tumors. BioData Min 2017;10:6.

16. Chang YC, Chan YC, Chang WM, et al. Feedback regulation of ALDOA activates the HIF- $1 \alpha / \mathrm{MMP} 9$ axis to promote lung cancer progression. Cancer Lett 2017;403:28-36.

17. Caspi M, Perry G, Skalka N, et al. Aldolase positively regulates of the canonical Wnt signaling pathway. Mol Cancer 2014;13:164.

18. Hwang SJ, Lee HW, Kim HR, et al. Ubiquitin-specific protease 4 controls metastatic potential through $\beta$-catenin stabilization in brain metastatic lung adenocarcinoma. Sci Rep 2016;6:21596.

19. Gonzalez DM, Medici D. Signaling mechanisms of the epithelial-mesenchymal transition. Sci Signal 2014;7:re8.

20. Stewart DJ. Wnt signaling pathway in non-small cell lung cancer. J Natl Cancer Inst 2014;106:djt356.

21. Lu L, Liu Q, Wang P, et al. MicroRNA-148b regulates tumor growth of non-small cell lung cancer through targeting MAPK/JNK pathway. BMC Cancer 2019;19:209.

22. Jiang W, Jia Q, Liu L, et al. S100B promotes the proliferation, migration and invasion of specific brain metastatic lung adenocarcinoma cell line. Cell Biochem Funct 2011;29:582-8.

23. Masuda C, Sugimoto M, Wakita D, et al. Bevacizumab suppresses the growth of established non-small-cell lung cancer brain metastases in a hematogenous brain metastasis model. Clin Exp Metastasis 2020;37:199-207.

24. Stoll LM, Johnson MW, Gabrielson E, et al. The utility of napsin-A in the identification of primary and metastatic lung adenocarcinoma among cytologically poorly differentiated carcinomas. Cancer Cytopathol 2010;118:441-9.

25. Siegel R, Ma J, Zou Z, et al. Cancer statistics, 2014. CA Cancer J Clin 2014;64:9-29.

26. He J, Li W, Li Y, et al. Construction of a prognostic model for lung adenocarcinoma based on bioinformatics analysis of metabolic genes. Transl Cancer Res 2020;9:3518-38.

27. Butnor KJ. Controversies and challenges in the histologic subtyping of lung adenocarcinoma. Transl Lung Cancer Res 2020;9:839-46.

28. Gerdan L, Segedin B, Nagy V, et al. Brain metastasis from non-small cell lung cancer (NSCLC): prognostic importance of the number of involved extracranial organs. Strahlenther Onkol 2014;190:64-7.

29. Girard N, Cozzone D, de Leotoing L, et al. Extra cost of brain metastases (BM) in patients with non-squamous non-small cell lung cancer (NSCLC): a French national hospital database analysis. ESMO Open 2018;3:e000414.

30. Pandey GK, Mitra S, Subhash S, et al. The risk-associated long noncoding RNA NBAT-1 controls neuroblastoma progression by regulating cell proliferation and neuronal differentiation. Cancer Cell 2014;26:722-37.

31. Gutschner T, Hämmerle $M$, Eissmann $M$, et al. The noncoding RNA MALAT1 is a critical regulator of the metastasis phenotype of lung cancer cells. Cancer Res 2013;73:1180-9.

32. Peng Z, Wang J, Shan B, et al. The long noncoding RNA LINC00312 induces lung adenocarcinoma migration and vasculogenic mimicry through directly binding YBX1. Mol Cancer 2018;17:167.

33. Jiang C, Zhao H, Yang B, et al. lnc-REG3G-3-1/miR-2153 p Promotes Brain Metastasis of Lung Adenocarcinoma by Regulating Leptin and SLC2A5. Front Oncol 2020;10:1344.

34. Zheng X, Zhang J, Fang T, et al. The long non-coding RNA PIK3CD-AS2 promotes lung adenocarcinoma progression via YBX1-mediated suppression of p53 pathway. Oncogenesis 2020;9:34.

35. Qi L, Liu F, Zhang F, et al. lncRNA NEAT1 competes against let-7a to contribute to non-small cell lung cancer proliferation and metastasis. Biomed Pharmacother 2018;103:1507-15.

36. Liu B, Pan CF, He ZC, et al. Long Noncoding RNALET Suppresses Tumor Growth and EMT in Lung Adenocarcinoma. Biomed Res Int 2016;2016:4693471.

37. Sheng K, Lu J, Zhao H. ELK1-induced upregulation of lncRNA HOXA10-AS promotes lung adenocarcinoma progression by increasing $W n t / \beta$-catenin signaling. Biochem Biophys Res Commun 2018;501:612-8.

38. Kopp F, Mendell JT. Functional Classification and Experimental Dissection of Long Noncoding RNAs. Cell 2018;172:393-407.

39. Chen LL. Linking Long Noncoding RNA Localization and Function. Trends Biochem Sci 2016;41:761-72.

40. Zhu J, Fu H, Wu Y, et al. Function of lncRNAs and approaches to lncRNA-protein interactions. Sci China Life Sci 2013;56:876-85.

41. Chang YC, Yang YC, Tien CP, et al. Roles of Aldolase Family Genes in Human Cancers and Diseases. Trends Endocrinol Metab 2018;29:549-59.

42. Gizak A, Wiśniewski J, Heron P, et al. Targeting a moonlighting function of aldolase induces apoptosis in cancer cells. Cell Death Dis 2019;10:712.

43. Cui K, Jin S, Du Y, et al. Long noncoding RNA DIO3OS 
interacts with miR-122 to promote proliferation and invasion of pancreatic cancer cells through upregulating ALDOA. Cancer Cell Int 2019;19:202.

44. Ji S, Zhang B, Liu J, et al. ALDOA functions as an oncogene in the highly metastatic pancreatic cancer. Cancer Lett 2016;374:127-35.

45. Dai L, Pan G, Liu X, et al. High expression of ALDOA and DDX5 are associated with poor prognosis in human colorectal cancer. Cancer Manag Res 2018;10:1799-806.

46. Li H, Zhang X, Jin Z, et al. MiR-122 Promotes the Development of Colon Cancer by Targeting ALDOA In Vitro. Technol Cancer Res Treat 2019;18:1533033819871300.

47. Xu Z, Liu G, Zhang M, et al. miR-122-5p Inhibits the Proliferation, Invasion and Growth of Bile Duct Carcinoma Cells by Targeting ALDOA. Cell Physiol Biochem 2018;48:2596-606.

48. Li J, Wang F, Gao H, et al. ALDOLASE A regulates invasion of bladder cancer cells via E-cadherin-EGFR signaling. J Cell Biochem 2019;120:13694-705.

49. Zhang C, Zhao LM, Wu H, et al. C/D-Box Snord105b Promotes Tumorigenesis in Gastric Cancer via ALDOA/ C-Myc Pathway. Cell Physiol Biochem 2018;45:2471-82.

50. Chang YC, Yang YF, Chiou J, et al. Nonenzymatic function of Aldolase A downregulates miR-145 to promote the Oct4/DUSP4/TRAF4 axis and the acquisition of lung cancer stemness. Cell Death Dis 2020;11:195.

51. Chang YC, Chiou J, Yang YF, et al. Therapeutic Targeting of Aldolase A Interactions Inhibits Lung Cancer Metastasis and Prolongs Survival. Cancer Res 2019;79:4754-66.

52. Fu H, Gao H, Qi X, et al. Aldolase A promotes proliferation and G1/S transition via the EGFR/MAPK pathway in non-small cell lung cancer. Cancer Commun

Cite this article as: Feng S, Liu H, Du P, Dong X, Pang Q, Guo H. Long non-coding RNA AC122108.1 promotes lung adenocarcinoma brain metastasis and progression through the Wnt/ $\beta$-catenin pathway by directly binding to aldolase A. Ann Transl Med 2021;9(23):1729. doi: 10.21037/atm-21-5707
(Lond) 2018;38:18.

53. Wang C, Xu J, Yuan D, et al. Exosomes carrying ALDOA and ALDH3A1 from irradiated lung cancer cells enhance migration and invasion of recipients by accelerating glycolysis. Mol Cell Biochem 2020;469:77-87.

54. Li H, Huang Y, Xiao Y. A pair-conformation-dependent scoring function for evaluating 3D RNA-protein complex structures. PLoS One 2017;12:e174662.

55. Guan $\mathrm{H}, \mathrm{Zhu} \mathrm{T}, \mathrm{Wu}$ S, et al. Long noncoding RNA LINC00673-v4 promotes aggressiveness of lung adenocarcinoma via activating $\mathrm{WNT} / \beta$-catenin signaling. Proc Natl Acad Sci U S A 2019;116:14019-28.

56. Kimelman $\mathrm{D}, \mathrm{Xu} W$. beta-catenin destruction complex: insights and questions from a structural perspective. Oncogene 2006;25:7482-91.

57. Angers S, Moon RT. Proximal events in Wnt signal transduction. Nat Rev Mol Cell Biol 2009;10:468-77.

58. Wang P, Xue Y, Han Y, et al. The STAT3-binding long noncoding RNA lnc-DC controls human dendritic cell differentiation. Science 2014;344:310-3.

59. Liu B, Sun L, Liu Q, et al. A cytoplasmic NF$\kappa \mathrm{B}$ interacting long noncoding RNA blocks IкB phosphorylation and suppresses breast cancer metastasis. Cancer Cell 2015;27:370-81.

60. Yang F, Zhang H, Mei Y, et al. Reciprocal regulation of HIF-1 $\alpha$ and lincRNA-p2 1 modulates the Warburg effect. Mol Cell 2014;53:88-100.

61. Gao Q, Zhu H, Dong L, et al. Integrated Proteogenomic Characterization of HBV-Related Hepatocellular Carcinoma. Cell 2019;179:561-77.e22.

(English Language Editor: L. Huleatt) 
Supplementary

Table S1 The relative sequences used for the PCR

\begin{tabular}{lll}
\hline Targeted gene & Forward primer 5'-3' & Reverse primer 5'-3' \\
\hline AC122108.1 & GGGTCAGTAGCAGTGGAACC & GTGGGCTCTGATGCAGAACT \\
Si-AC122108.1 & GCCAAUAUGUCUCUAGAAATT & UUUCUAGAGACAUAUUGGCTT \\
ALDOA & GAGGCGTCCATCAACCTCAA & GCAGCCTTCAGGTTCTCCTT \\
GAPDH & TGACTTCAACAGCGACACCCA & CACCCTGTTGCTGTAGCCAAA \\
\hline
\end{tabular}

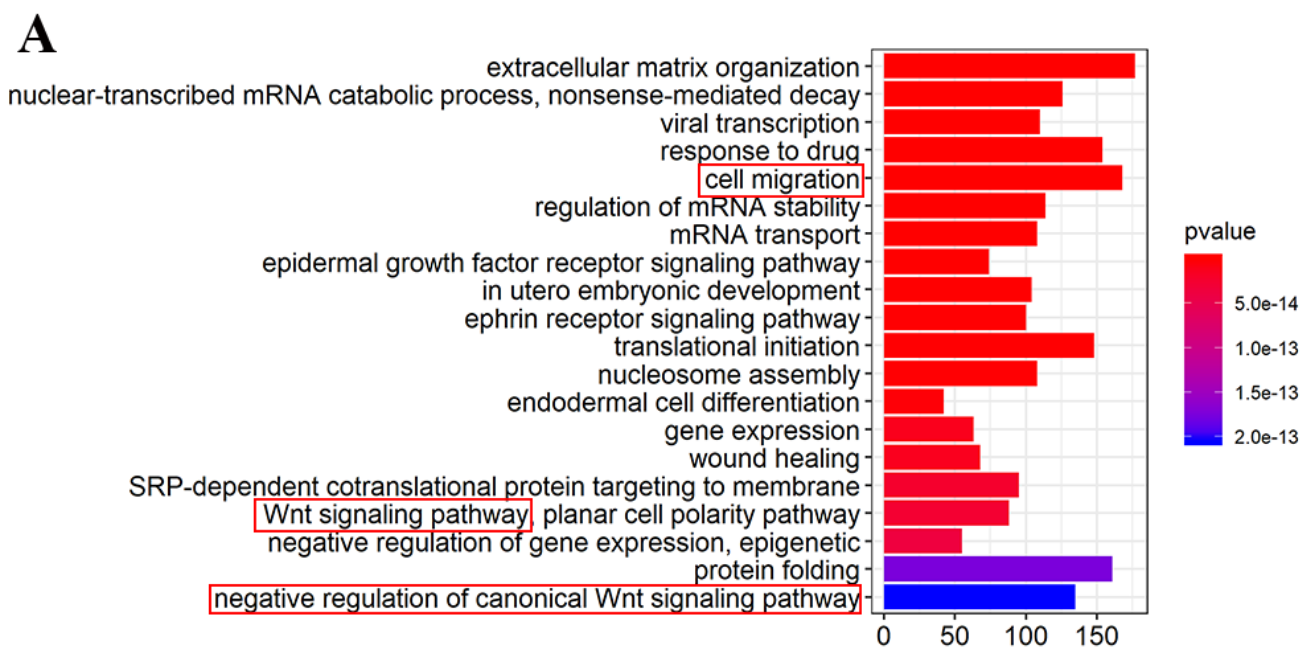

B

Bladder cancerMetabolism of xenobiotics by cytochrome P450 Glycosaminoglycan biosynthesis - chondroitin sulfate / dermatan sulfate Hippo signaling pathway -multiple species Drug metabolism - cytochrome P450 Glutathione metabolism Glycosphingolipid biosynthesis - globo and isoglobo series Mucin type O-glycan biosynthesis Basal transcription factors Pathogenic Escherichia coli infection Cocaine addiction Thyroid cancer Drug metabolism - other enzymes Vitamin B6 metabolism Pentose phosphate pathway Ferroptosis Steroid biosynthesis Retinol metabolism Pentose and glucuronate interconversions Non-homologous end-joining

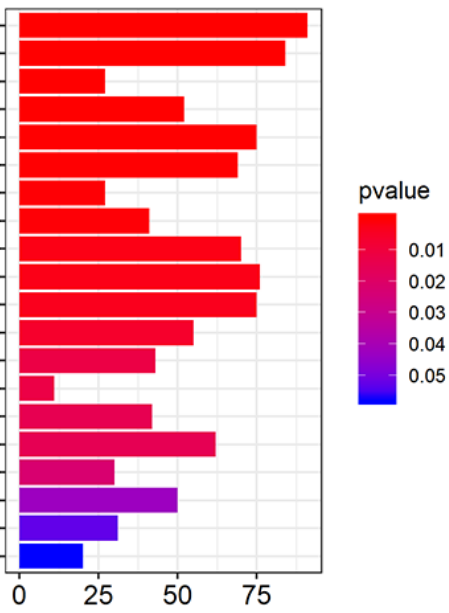

Figure S1 The transcriptional changes were displayed at the overall level by the GO and KEGG classification analysis. (A) The GO significant enrichment analysis showed that these differently expressed genes are mainly involved in the Wnt signaling pathway. (B) The KEGG significant enrichment analysis showed that these differently expressed genes are mainly involved in cancers and the pentose phosphate pathway. 